\title{
A Shannon wavelet method for pricing foreign exchange options under the Heston multi-factor CIR model *
}

\author{
Edouard Berthe ${ }^{\dagger}$
}

Duy-Minh Dang $\ddagger$

Luis Ortiz-Gracia $\S$

\begin{abstract}
We present a robust and highly efficient Shannon wavelet pricing method for plain-vanilla foreign exchange European options under the jump-extended Heston model with multi-factor CIR interest rate dynamics. Under a Monte Carlo and partial differential equation hybrid computational framework, the option price can be expressed as an expectation, conditional on the variance factor, of a convolution product that involves the densities of the time-integrated domestic and foreign multi-factor CIR interest rate processes. We propose an efficient treatment to this convolution product that effectively results in a significant dimension reduction, from two multi-factor interest rate processes to only a single-factor process. By means of a state-of-the-art Shannon wavelet inverse Fourier technique, the resulting convolution product is approximated analytically and the conditional expectation can be computed very efficiently. We develop sharp approximation error bounds for the option price and hedging parameters. Numerical experiments confirm the robustness and efficiency of the method.
\end{abstract}

\section{Introduction}

In the current era of wildly fluctuating exchange rates, foreign exchange (FX) financial contracts, i.e. derivatives, are of enormous practical importance. There has been great interest in modelling FX derivatives using four factor jump-diffusion models 1 See Ahlip et al. (2017); Ahlip and Rutkowski (2013, 2015); Cozma et al. (2018); Cozma and Reisinger (2017) among many other publications. Typically, in these models, the spot FX rate and its variance follow a jump-extension of the Heston model (Heston, 1993), while the domestic and foreign interest rates follow the one-factor Hull-White or CoxIngersoll-Ross (CIR) dynamics (Cox et al., 1985a; Hull and White, 1993). From a risk management point of view, FX models with jumps are useful, as they permit us to explore the effects of severe market crashes on FX rates. This is potentially important for long-dated (maturities of 20 years or more) FX derivatives embedded with popular early exercise contract features, such as Bermudan cancelable, knock-out, and Target Redemption (Clark, 2011; Qu, 2016).

Despite of their popularity, one-factor interest rate models suffer from a well-known limitation, namely their inability to accurately capture de-correlations, i.e. non-perfect correlations, between rates for different maturities. This issue is particularly crucial in modelling of (long-dated) FX interest rate derivatives, such as Power-Reverse Dual-Currency (PRDC) swaps and FX Target Redemption Notes, due to their strong dependence on movements in both domestic and foreign interest rates (Caps, 2007; Col et al., 2013; Dang et al., 2014, 2010, 2015a; Mallo, 2010; Piterbarg, 2006; Sippel and Ohkoshi,

${ }^{*}$ This research was supported in part by the Spanish Ministry of Economy and Competitiveness for funding under grants ECO2016-76203-C2-2 and MTM2016-76420-P (MINECO/FEDER, UE).

†École Centrale Paris and The University of Queensland email: berthe.ed@gmail.com

${ }^{\ddagger}$ School of Mathematics and Physics, The University of Queensland, St Lucia, Brisbane 4072, Australia, email: duyminh.dang@uq.edu.au

${ }^{\S}$ Universitat de Barcelona School of Economics, Faculty of Economics and Business, University of Barcelona, John M. Keynes 1-11, 08034 Barcelona, Spain, email: luis.ortiz-gracia@ub.edu

${ }^{1} \mathrm{~A}$ stochastic factor is a Brownian motion. 
2002). These derivatives have become increasingly important and are traded in large quantities in Over-the-Counter markets. In fact, it is suggested in the interest rate literature that, in order to sufficiently capture de-correlations in the rates, multi-factor interest rate dynamics should be used (Brigo and Mercurio, 2006; Jamshidian and Zhu, 1997; Rebonato, 1998).

The use of multi-factor Gaussian interest rates dynamics in option pricing is recently explored extensively in Dang (2017); Dang et al. (2015b, 2017); Dang and Ortiz-Gracia (2018). This paper is a continuation of these first steps towards a more realistic modelling framework for FX derivatives across a wide range of maturities and/or contract features. Specifically, in this paper, we consider a general FX model in which interest short rates follow multi-factor CIR dynamics, whereas the spot FX rate and its instantaneous variance is jointly governed by a jump-extended Heston model. Typically, multi-factor CIR dynamics for the interest rates would allow for a closer match of skewed market implied distributions of interest rates in a wide range of maturities than their multi-factor Gaussian counterparts (Brigo and Mercurio, 2006).

In general, for model calibration purposes, highly efficient pricing methods for plain-vanilla $\mathrm{Eu}-$ ropean options are typically required. Since a closed-form solution for plain-vanilla European FX options is not available for the model considered in this work, an efficient numerical pricing method must be developed for these derivatives. However, the mathematical and computational challenge posed by this model is particularly significant, because in this case we need to efficiently handle a convolution product that involves two unknown densities of the time-integrated domestic and foreign (multi-factor) CIR interest rate processes. Due to these reasons, in this paper, we primarily focus on the development of highly efficient numerical methods for plain-vanilla European FX options, leaving model calibration to future work.

In option pricing, state-of-the-art numerical integration based methods, such as the COS method of Fang and Oosterlee (2008) or the Shannon Wavelet Inverse Fourier Technique (SWIFT) proposed in Ortiz-Gracia and Oosterlee (2016), if applicable, are significantly more efficient than Monte-Carlo or partial differential equation (PDE). These methods typically require knowing a closed-form expression for the characteristic function of the underlying stochastic process so that the corresponding density function can be recovered. However, for the type of general models under investigation, as well as for many other interesting models, such a closed-form expression for the characteristic function of the underlying process is difficult, perhaps impossible, to obtain.

This paper aims to further extend the applicabilities of these state-of-the-art numerical integration methods to the above-mentioned general jump-diffusion FX model. We use the SWIFT method, due to the established robustness of Shannon wavelets in option pricing, as demonstrated in a number of works, such as Colldeforns-Papiol et al. (2017); Maree et al. (2017); Ortiz-Gracia and Oosterlee (2016). The proposed SWIFT-based method is developed within the hybrid MC-PDE computational framework put forward in Dang et al. (2015b, 2017). This framework generally allows to express the option price as the expectation of the unique solution to an associated conditional Partial IntegroDifferential Equation (PIDE). This solution is cast in the form of a multi-dimensional convolution product that involves densities of the time-integrated domestic and foreign interest rate processes. These densities are unknown for multi-factor CIR dynamics, and hence must be approximated. This results in a very complex convolution product that must be handled in a highly efficient manner. Such substantial mathematical and computational challenge differentiates this work from previous ones on multi-factor Gaussian interest rates (Dang et al., 2017; Dang and Ortiz-Gracia, 2018), since in the latter case, the density of the time-integrated Gaussian process is known in closed form.

The main contributions of paper can be summarized as follows.

- By means of the SWIFT method, we propose an efficient treatment of the above-mentioned complex convolution product that effectively results in a significant dimension reduction from two multi-factor CIR interest rate processes, to only a single-factor CIR dynamics. Moreover, this dimension reduction is independent of the total number of interest rate factors in the model. 
- We recover the classical FX option formulas in Garman and Kohlhagen (1983) for the solution of the conditional PIDE when using effective constant domestic and foreign risk-free rates.

- The (outer) expectation can be expressed as a two-dimensional integral that involves only (i) the value of the variance at the terminal time, and (ii) the time-integrated variance process conditional on this value. This two-dimensional integral can be further reduced to the evaluation of just a single integral that involves only the density of the terminal variance value, thanks to the excellent approximation properties of Shannon wavelets.

- Extensive numerical experiments confirm the robustness and significant efficiency of the proposed pricing technique, while the computational complexity remains independent of the number of stochastic factors in the model.

The remainder of the paper is organized as follows. We start by introducing a general pricing model and reviewing the dimension reduction framework in Sections 2 and 3 , respectively. Section 4 discusses the development of an efficient SWIFT-based numerical technique for the solution to the conditional PIDE. In Section 5, we present the formulas for the solution of the conditional PIDE for the case of call and put options. The outer expectation is treated in Section [6. Section 7 develops the error analysis. In Section 8, we present several numerical results to illustrate the method's robustness, error bounds, and efficiency. Section 9 concludes the paper and outlines possible future work.

\section{Model}

We consider an (international) economy consisting of two markets (currencies) indexed by $i \in\{d, f\}$, where " $d$ " and " $f$ " stand for the domestic and foreign markets, respectively. We consider a complete probability space $\left(\Omega, \mathcal{F},\left\{\mathcal{F}_{t}\right\}_{t \geq 0}, \mathbb{Q}\right)$, with sample space $\Omega$, sigma-algebra $\mathcal{F}$, filtration $\left\{\mathcal{F}_{t}\right\}_{t \geq 0}$, and risk-neutral measure $\mathbb{Q}$ defined on $\mathcal{F}$. We denote by $\mathbb{E}$ the expectation taken under $\mathbb{Q}$ measure. We denote by $S(t)$ the spot FX rate, which is defined as the number of units of domestic currency per one unit of foreign currency. Let the spot FX rate $S(t)$, its instantaneous variance $\nu(t)$, and the two short rates $r_{d}(t)$ and $r_{f}(t)$ be governed by the following SDEs under the measure $\mathbb{Q}$ :

$$
\begin{aligned}
\frac{\mathrm{d} S(t)}{S\left(t^{-}\right)}= & \left(r_{d}(t)-r_{f}(t)-\lambda \delta\right) \mathrm{d} t+\sqrt{\nu(t)} \mathrm{d} W_{s}(t)+\mathrm{d} J(t), \\
r_{d}(t)= & \sum_{i=1}^{p_{d}} X_{i}(t) \\
& \quad \text { with } \mathrm{d} X_{i}(t)=\kappa_{d_{i}}\left(\theta_{d_{i}}-X_{i}(t)\right) \mathrm{d} t+\sigma_{d_{i}} \sqrt{X_{i}(t)} \mathrm{d} W_{d_{i}}(t), \\
r_{f}(t)= & \sum_{j=1}^{p_{f}} Y_{j}(t), \\
& \text { with } \mathrm{d} Y_{j}(t)=\kappa_{f_{j}}\left(\theta_{f_{j}}-Y_{j}(t)\right) \mathrm{d} t+\sigma_{f_{j}} \sqrt{Y_{j}(t)} \mathrm{d} W_{f_{j}}(t)-\rho_{s, f_{j}} \sigma_{f_{j}} \sqrt{\nu(t)} \mathrm{d} t, \\
\mathrm{~d} \nu(t)= & \kappa_{\nu}(\bar{\nu}-\nu(t)) \mathrm{d} t+\sigma_{\nu} \sqrt{\nu(t)} \mathrm{d} W_{\nu}(t) .
\end{aligned}
$$

We work under the following assumptions for model (2.1).

- Processes $W_{s}(t)$ and $W_{\nu}(t)$ are correlated Brownian motions (BMs) with a constant correlation coefficient $\rho \in[-1,1]$. As we will illustrate in a later section, the assumption on a constant correlation $\rho$ is indeed crucial to the method. Processes $W_{s}(t)$ and $W_{\nu}(t)$ are independent of processes $W_{d_{i}}(t), i=1, \ldots, p_{d}$, as well as of processes $W_{f_{j}}(t), j=1, \ldots, p_{f}$. Processes $W_{d_{i}}(t), i=1, \ldots, p_{d}$, and $W_{f_{j}}(t), j=1, \ldots, p_{f}$, are pairwise independent. As we will argue in what follows, this assumption is also crucial for analyticity of the method. We note that the 
independence assumption between factors of a multi-factor CIR interest rate process appears to be a standard assumption in the literature on the subject (see, e.g. Chen and Scott (1992, 2003); Nawalkha et al. (2007)).

- The process $J(t)=\sum_{j=1}^{\pi(t)}\left(x_{j}-1\right)$ is a compound Poisson process. Specifically, $\pi(t)$ is a Poisson process with a constant finite jump intensity $\lambda>0$, and $x_{j}, j=1,2, \ldots$, are independent and identically distributed (i.i.d.) positive random variables representing the jump amplitude, and having the density $\chi(\cdot)$. Several popular cases for $\chi(\cdot)$ are (i) the log-normal distribution given in Merton (1976), and (ii) the log-double-exponential distribution given in Kou (2002). When a jump occurs at time $t$, we have $S(t)=x S\left(t^{-}\right)$, where $t^{-}$is the instant of time just before the time $t$. In (2.1),$\delta=\mathbb{E}[x-1]$ represents the expected percentage change in the spot FX rate.

- The Poisson process $\pi(t)$, and the sequence of random variables $\left\{x_{j}\right\}_{j=1}^{\infty}$ are mutually independent, as well as independent of the BMs $W_{s}(t), W_{d_{i}}(t), i=1, \ldots, n, W_{f_{i}}(t), i=1, \ldots, l$, and $W_{\nu}(t)$.

- The quantities $\kappa_{d_{i}}, \sigma_{d_{i}}, i=1, \ldots, p_{d}, p_{d} \geq 1, \kappa_{f_{j}}$, and $\sigma_{f_{j}}, j=1, \ldots, p_{f}, p_{f} \geq 1$, are strictly positive constants.

For use later in the paper, we write $\left(\begin{array}{l}W_{s} \\ W_{\nu}\end{array}\right)=\left(\begin{array}{cc}\sqrt{1-\rho^{2}} & \rho \\ 0 & 1\end{array}\right)\left(\begin{array}{l}W_{1} \\ W_{2}\end{array}\right)$, where $W_{1}$ and $W_{2}$ are independent Brownian motions, and $\rho$ is the constant correlation between $W_{s}$ and $W_{\nu}$. We denote by $V(S(t), t, \cdot) \equiv V\left(S(t), t, r_{d}(t), r_{f}(t), \nu(t)\right)$ the price at time $t$ of a plain-vanilla European option under the model (2.1) with payoff $\Phi(S(T))$. We further assume that the payoff $\Phi(x)$ is a continuous function of its argument having at most polynomial (sub-exponential) growth. This condition is satisfied in the case of call and put options, where $\Phi(S(T))=\max (S(T)-K, 0)$ and $\Phi(S(T))=\max (K-S(T), 0)$, respectively. Here, $K$ is the strike of the option.

While model calibration to existing market data is not a focus of this paper, we briefly discuss how this can be done, without going into detail. The constant correlation $\rho$ can be obtained from historical data. The calibration procedure can be performed in two stages. In the first stage, the parameters for the multi-factor short rate processes are determined, independently of the FX part (Brigo and Mercurio, 2006). In the second stage, the calibrated short rate processes are included in the Heston model, and the remaining parameters are determined. In this stage, the calibration can be expressed as a nonlinear least-squares problem. We refer the reader to Cui et al. (2017) for a summary of existing numerical optimization methods to solve this problem. We emphasize that highly efficient pricing methods, which is the focus of the present paper, are crucial for the second stage.

\section{A hybrid MC-PDE/PIDE approach}

\subsection{General framework}

In the first step of the proposed approach, we follow the hybrid MC-PDE/PIDE approach in Dang et al. (2015b, 2017). Below, we briefly summarize the main steps of this framework. The reader is referred to Dang et al. (2015b, 2017) for detailed discussions and relevant proofs.

Using standard arbitrage theory (Delbaen and Schachermaver, 1994), and the "tower property" of the conditional expectation, the option price under the general model (2.1) can be expressed as the two-level nested expectation

$$
V(S(0), 0, \cdot)=\mathbb{E}\left[\mathrm{e}^{-\int_{0}^{T} r_{d}(t) \mathrm{d} t} \Phi(S(T))\right]=\mathbb{E}\left[\mathbb{E}\left[\mathrm{e}^{-\int_{0}^{T} r_{d}(t) \mathrm{d} t} \Phi(S(T)) \mid\left\{W_{2}(\tau)\right\}\right]\right] .
$$

Here, $\left\{W_{2}(\tau)\right\} \equiv\left\{W_{2}(\tau ; 0 \leq \tau \leq T)\right\}$ denotes the filtration generated by the corresponding BM. Under certain regularity conditions, which are satisfied in the present case, by the Feynman-Kac theorem for 
jump-diffusion processes (Cont and Tankov, 2004), the inner expectation of (3.1) can be shown to be equal to the unique solution to an associated (conditional) PIDE (Dang et al., 2017)

To solve the conditional PIDE, we first transform it into the Fourier space to obtain an ordinary differential equation in terms of a transformed option price. This ordinary differential equation can then be easily solved in closed-form from maturity $t=T$ to time $t=0$ to obtain the transformed solution of the conditional PIDE at time $t=0$. Let

$$
z=\ln (x), \quad \phi(z)=\Phi\left(\mathrm{e}^{z}\right), \quad v(z, t, \cdot)=V(x, t, \cdot),
$$

and we denote by $\hat{f}(\xi)$ the Fourier transform of a generic function $f$, i.e. $\hat{f}(\xi)=\frac{1}{\sqrt{2 \pi}} \int_{\mathbb{R}} e^{-i \xi x} f(x) \mathrm{d} x$. It can be shown that (Dang et al., 2015b, 2017)

$$
\hat{v}(0, \xi)=\mathbb{E}\left[\hat{\phi}(\xi) \mathrm{e}^{-G \xi^{2}+i \xi F-\lambda T+\lambda T \Gamma(\xi)+(i \xi-1) \int_{0}^{T} r_{d}(t) \mathrm{d} t-i \xi \int_{0}^{T} r_{f}(t) \mathrm{d} t}\right],
$$

where

$$
G=\frac{1-\rho^{2}}{2} \int_{0}^{T} \nu(t) \mathrm{d} t, \quad F=-\frac{1}{2} \int_{0}^{T} \nu(t) \mathrm{d} t+\rho \int_{0}^{T} \sqrt{\nu(t)} \mathrm{d} W_{2}(s)-\lambda \delta T,
$$

and $\Gamma(\xi)$ is the characteristic function of $\ln (y)$, i.e. the log of the jump amplitude $y$. We emphasize that, while $G$ and $F$ are stochastic, they depend only on the variance factor $\nu(t)$. Furthermore, the characteristic function $\Gamma(\xi)$ is known for popular jump models, such as when $\ln (y)$ follows a normal (Merton, 1976) or a double-exponential distribution (Kou, 2002).

The last step is to invert (3.3). First, we apply iterated conditional expectation to obtain

$$
\begin{aligned}
\hat{v}(0, \xi) & =\mathbb{E}\left[\mathbb{E}\left[\hat{\phi}(\xi) e^{-G \xi^{2}+i \xi F-\lambda T+\lambda T \Gamma(\xi)+(i \xi-1) \int_{0}^{T} r_{d}(t) \mathrm{d} t-i \xi \int_{0}^{T} r_{f}(t) \mathrm{d} t} \mid\left\{W_{2}(\tau)\right\}\right]\right] \\
& =\mathbb{E}\left[\hat{\phi}(\xi) e^{-G \xi^{2}+i \xi F-\lambda T+\lambda T \Gamma(\xi)} \mathbb{E}\left[e^{(i \xi-1) \int_{0}^{T} r_{d}(t) \mathrm{d} t}\right] \mathbb{E}\left[e^{-i \xi \int_{0}^{T} r_{f}(t) \mathrm{d} t}\right]\right] \\
& =\mathbb{E}\left[\hat{\phi}(\xi) \mathrm{e}^{-G \xi^{2}+i \xi F-\lambda T+\lambda T \Gamma(\xi)} \Psi_{d}(\xi+i) \Psi_{f}(-\xi)\right]
\end{aligned}
$$

where $\Psi_{d}(\cdot)$ and $\Psi_{f}(\cdot)$ respectively are the characteristic functions of the time-integrated domestic and foreign interest rate processes. The second equality in (3.5) is the result of the independency between the domestic, as well as foreign, rate and the variance. Furthermore, $\Psi_{d}(\cdot)$ and $\Psi_{f}(\cdot)$ can be obtained in closed-form using an expression for the characteristic function of the time-integrated CIR process available in Dufresne (2001). Specifically, we have

$$
\begin{aligned}
& \Psi_{d}(\xi)=\mathbb{E}\left[\mathrm{e}^{i \xi \int_{0}^{T} r_{d}(t) \mathrm{d} t}\right]=\mathbb{E}\left[\mathrm{e}^{\left.i \xi \sum_{j=1}^{p_{d}} \int_{0}^{T} X_{j}(t) \mathrm{d} t\right]}=\prod_{j=1}^{p_{d}} \mathbb{E}\left[\mathrm{e}^{\left.i \xi \int_{0}^{T} X_{j}(t) \mathrm{d} t\right]}=\prod_{j=1}^{p_{d}} \Psi_{d_{j}}(\xi)\right.\right. \\
& =\prod_{j=1}^{p_{d}}\left(\frac{\mathrm{e}^{\frac{\kappa_{d_{j}} T}{2}}}{\cosh \left(\frac{\gamma_{d_{j}}(\xi) T}{2}\right)+\frac{\kappa_{d_{j}}}{\gamma_{d_{j}}(\xi)} \sinh \left(\frac{\gamma_{d_{j}}(\xi) T}{2}\right)}\right)^{\frac{2 \kappa_{d_{j}} \theta_{d_{j}}}{\sigma_{d_{j}}^{2}}} \exp \left(\frac{2 i \xi X_{j}(0) \sinh \left(\frac{\gamma_{d_{j}}(\xi) T}{2}\right)}{\gamma_{d_{j}}(\xi) \cosh \left(\frac{\gamma_{d_{j}}(\xi) T}{2}\right)+\kappa_{d_{j}} \sinh \left(\frac{\gamma_{d_{j}}(\xi) T}{2}\right)}\right),
\end{aligned}
$$

where $\gamma_{d_{j}}(\xi)=\sqrt{\kappa_{d_{j}}^{2}-2 i \sigma_{d_{j}}^{2} \xi}$, and the third equality comes from the independence of the interest rate factors. Here, we note that $\mathbb{E}\left[\mathrm{e}^{i \xi \int_{0}^{T} X_{j}(t) \mathrm{d} t}\right]=\Psi_{d_{j}}(\xi)$ is the characteristic function of the timeintegrated CIR process and its closed-form expression is available in Dufresne (2001). A similar expression can be found for $\Psi_{f}(\xi)$.

We emphasize that it would not have been possible to obtain the simple expression (3.6) for $\Psi_{d}(\xi)$ (resp. $\Psi_{f}(\xi)$ ), if the factors of the domestic (resp. foreign) interest rate dynamics are not independent. 
We note that, as mentioned earlier in Section 2, this independence assumption appears to be a standard assumption in the literature on multi-factor CIR interest rate processes (see, e.g. Chen and Scott (1992, 2003); Nawalkha et al. (2007)). Furthermore, if the correlation between $S$ and $r_{d}$ (or between $S$ and $r_{f}$ ) is non-zero, in (3.3), we would have had quantities of the form $e^{i \xi \int_{0}^{T} \sqrt{\nu(t)} \mathrm{d} W_{d_{j}}(t)}, j=1, \ldots, p_{d}$ (or $\left.e^{i \xi \int_{0}^{T} \sqrt{\nu(t)} \mathrm{d} W_{f_{j}}(t)}, j=1, \ldots, p_{f}\right)$, and hence the iterated conditional expectation used in (3.5) would not have resulted in $\mathbb{E}\left[e^{(i \xi-1) \int_{0}^{T} r_{d}(t) \mathrm{d} t}\right]$ and $\mathbb{E}\left[e^{-i \xi \int_{0}^{T} r_{f}(t) \mathrm{d} t}\right]$ being factored out.

\subsection{Two treatments of $\Psi_{d}(\xi+i) \Psi_{f}(-\xi)$}

To obtain the option price, we need to apply the inverse Fourier transform to (3.5). We now propose two different treatments for the term $\Psi_{d}(\xi+i) \Psi_{f}(-\xi)$ in (3.5). In the first treatment, we handle $\Psi_{d}(\xi+i)$ and $\Psi_{f}(-\xi)$ separately when the inverse Fourier transform is applied. This will result in a convolution product of two densities for the time-integrated domestic and foreign interest rate processes, and each density needs to be recovered separately using numerical methods. We refer to this treatment as the "two-density" one. The other treatment is motivated by the independence between the domestic and foreign interest rates. Specifically, we treat $\Psi_{d}(\xi+i) \Psi_{f}(-\xi)$ as a single function of $\xi$ when the inverse Fourier transform is applied. This will result in only one function to be recovered by numerical methods in the next step. We hereafter refer to this treatment as the "combined-density" one.

We denote by $\mathcal{F}^{-1}(\cdot)$ the inverse Fourier transform operator. With respect to the "two-density" treatment, by applying the inverse Fourier transform, on (3.5), together with the convolution theorem and Fubini's theorem, we obtain

$$
\begin{aligned}
v(0, z) & =\mathbb{E}\left[\phi * \mathcal{F}^{-1}\left(\xi \mapsto \mathrm{e}^{-G \xi^{2}+i \xi F-\lambda T+\lambda T \Gamma(\xi)}\right) * \mathcal{F}^{-1}\left(\xi \mapsto \Psi_{d}(\xi+i)\right) * \mathcal{F}^{-1}\left(\xi \mapsto \Psi_{f}(-\xi)\right)\right](z) \\
& =\mathbb{E}\left[\phi * \mathcal{F}^{-1}\left(\xi \mapsto \mathrm{e}^{-G \xi^{2}+i \xi F-\lambda T+\lambda T \Gamma(\xi)}\right) *\left(t \mapsto \mathrm{e}^{t} \mathcal{F}^{-1} \Psi_{d}(t)\right) * \mathcal{F}^{-1} \Psi_{f}(t)\right](z) \\
& =2 \pi \mathbb{E}\left[\phi * \mathcal{F}^{-1}\left(\xi \mapsto \mathrm{e}^{-G \xi^{2}+i \xi F-\lambda T+\lambda T \Gamma(\xi)}\right) * \mathrm{e}^{t} f_{d}(-t) * f_{f}(t)\right](z),
\end{aligned}
$$

where ${ }^{*}$ denotes the convolution product, and $f_{d}(\cdot)$ and $f_{f}(\cdot)$ respectively are the densities of the time-integrated domestic and foreign interest rate processes. Here, the second equality comes from the shifting theorems of Fourier transforms, and the third equality comes from the fact that the characteristic function of any random variable can be expressed as an inverse Fourier transform of the density function of that variable.

With respect to the "combined-density" treatment, we first define

$$
\Psi_{c}(\xi)=\Psi_{d}(\xi+i) \Psi_{f}(-\xi)
$$

Then, following the same inverse Fourier transform technique as above, we have

$$
\begin{aligned}
v(0, z) & =\mathbb{E}\left[\phi * \mathcal{F}^{-1}\left(\xi \mapsto \mathrm{e}^{-G \xi^{2}+i \xi F-\lambda T+\lambda T \Gamma(\xi)}\right) * \mathcal{F}^{-1} \Psi_{c}\right](z) \\
& =\mathbb{E}\left[\phi * \mathcal{F}^{-1}\left(\xi \mapsto \mathrm{e}^{-G \xi^{2}+i \xi F-\lambda T+\lambda T \Gamma(\xi)}\right) *\left(t \mapsto f_{c}(-t)\right)\right](z),
\end{aligned}
$$

where

$$
f_{c}=\frac{1}{\sqrt{2 \pi}} \mathcal{F}^{-1} \Psi_{c}=\sqrt{2 \pi}\left(t \mapsto e^{-t} f_{d}(t)\right) *\left(t \mapsto f_{f}(-t)\right) .
$$

Therefore, $f_{c}(\cdot)$ can be interpreted as a convolution product between the densities of the timeintegrated domestic rate and the time-integrated foreign rate (symetrised).

We note that $f_{d}(\cdot), f_{f}(\cdot)$, and $f_{c}(\cdot)$, are not known in closed form, and hence numerical methods must be used to approximate them. This is the focus of the next section. In the remainder of this section, we will focus on $\mathcal{F}^{-1}\left(\xi \mapsto \mathrm{e}^{-G \xi^{2}+i \xi F-\lambda T+\lambda T \Gamma(\xi)}\right)$. For illustration purposes, we assume that 
the $\log$ of the jump amplitude $\ln (y) \sim \operatorname{Normal}\left(\tilde{\mu}, \tilde{\sigma}^{2}\right)$ (Merton, 1976). That is, the characteristic function $\Gamma(\xi)$ is $\Gamma(\xi)=\mathrm{e}^{i \tilde{\mu} \xi-\frac{1}{2} \tilde{\sigma}^{2} \xi^{2}}$. To deal with this term, we expand the term $\mathrm{e}^{\lambda T \Gamma(\xi)}$ in a Taylor series. Simple algebra shows that

$$
\begin{aligned}
\mathcal{F}^{-1}\left(\xi \mapsto \mathrm{e}^{-G \xi^{2}+i \xi F-\lambda T+\lambda T \Gamma(\xi)}\right)(z) & =\frac{1}{\sqrt{2 \pi}} \sum_{n=0}^{\infty} \frac{(\lambda T)^{n}}{n !} \int_{-\infty}^{+\infty} \mathrm{e}^{-G \xi^{2}+i \xi(z+F)-\lambda T} \mathrm{e}^{n i \tilde{\mu} \xi-\frac{1}{2} n \tilde{\sigma}^{2} \xi^{2}} \mathrm{~d} \xi \\
& =\sum_{n=0}^{\infty} \frac{(\lambda T)^{n}}{n !} \frac{1}{\sqrt{2 G+n \tilde{\sigma}^{2}}} e^{\left(-\lambda T-\frac{(z+F+n \tilde{\mu})^{2}}{2\left(2 G+n \tilde{\sigma}^{2}\right)}\right)}
\end{aligned}
$$

We conclude this section by noting that when the log of the jump amplitude follows the double exponential model proposed in Kou (2002), it is possible to obtain an analytical expression for $\mathcal{F}^{-1}\left(\xi \mapsto \mathrm{e}^{-G \xi^{2}+i \xi F-\lambda T+\lambda T \Gamma(\xi)}\right)$, although the expression is much more complex (Dang et al., 2017).

\section{Shannon wavelets}

In this section, we focus on recovering the unknown densities $f_{d}(\cdot)$ and $f_{f}(\cdot)$ (the "two-density" treatment, as well as $f_{c}(\cdot)$ (the "combined-density" treatment), via the SWIFT method developed by Ortiz-Gracia and Oosterlee (2016). For sake of completeness, we give below a brief introduction in Section 4.1 about multi-resolution analysis and Shannon wavelets.

\subsection{Multi-resolution analysis and Shannon wavelets}

Consider the space of square-integrable functions, denoted by $L^{2}(\mathbb{R})$, where

$$
L^{2}(\mathbb{R})=\left\{f: \int_{-\infty}^{+\infty}|f(x)|^{2} \mathrm{~d} x<\infty\right\} .
$$

A general structure for wavelets in $L^{2}(\mathbb{R})$ is called a multi-resolution analysis. We start with a family of closed nested subspaces in $L^{2}(\mathbb{R})$

$$
\ldots \subset \mathcal{V}_{-2} \subset \mathcal{V}_{-1} \subset \mathcal{V}_{0} \subset \mathcal{V}_{1} \subset \mathcal{V}_{2} \subset \ldots
$$

where

$$
\bigcap_{m \in \mathbb{Z}} \mathcal{V}_{m}=\{0\}, \quad \overline{\bigcup_{m \in \mathbb{Z}} \mathcal{V}_{m}}=L^{2}(\mathbb{R})
$$

and

$$
f(x) \in \mathcal{V}_{m} \Longleftrightarrow f(2 x) \in \mathcal{V}_{m+1}
$$

If these conditions are met, then there exists a function $\varphi \in \mathcal{V}_{0}$ that generates an orthonormal basis, denoted by $\left\{\varphi_{m, k}\right\}_{k \in \mathbb{Z}}$, for each $\mathcal{V}_{m}$ subspace, where

$$
\varphi_{m, k}(x)=2^{m / 2} \varphi\left(2^{m} x-k\right) .
$$

The function $\varphi(\cdot)$ is usually referred to as the scaling function or father wavelet.

For any $f \in L^{2}(\mathbb{R})$, a projection map of $L^{2}(\mathbb{R})$ onto $\mathcal{V}_{m}$, denoted by $\mathcal{P}_{m}: L^{2}(\mathbb{R}) \rightarrow \mathcal{V}_{m}$, is defined by means of

$$
\mathcal{P}_{m} f(x)=\sum_{k \in \mathbb{Z}} c_{m, k} \varphi_{m, k}(x)
$$

Here,

$$
c_{m, k}=\left\langle f, \varphi_{m, k}\right\rangle,
$$

where $\langle f, g\rangle=\int_{\mathbb{R}} f(x) \overline{g(x)} \mathrm{d} x$ denotes the inner product in $L^{2}(\mathbb{R})$, with $\overline{g(\cdot)}$ being the complex conjugation of $g(\cdot)$, and $\mathcal{P}_{m} f$ converges to $f$ in $L^{2}(\mathbb{R})$, i.e. $\left\|f-\mathcal{P}_{m} f\right\|_{2} \rightarrow 0$, when $m \rightarrow+\infty$. 
294

Considering higher $m$ values (i.e. when more terms are used), the accuracy of the truncated series representation of the function $f$ improves. As opposed to Fourier series, a key fact regarding the use of wavelets is that wavelets can be moved (by means of the $k$ value), stretched or compressed (by means of the $m$ value) to accurately represent the local properties of a function.

Shannon wavelets (Cattani, 2008) represent the real part of the so-called harmonic wavelets. They have a slow decay in the time domain but a very sharp compact support in the frequency, i.e. Fourier, domain. A set of Shannon scaling functions $\varphi_{m, k}(\cdot)$ in the subspace $\mathcal{V}_{m}$ is defined as

$$
\varphi_{m, k}(x)=2^{m / 2} \frac{\sin \left(\pi\left(2^{m} x-k\right)\right)}{\pi\left(2^{m} x-k\right)}=2^{m / 2} \varphi\left(2^{m} x-k\right), \quad k \in \mathbb{Z},
$$

where

$$
\varphi(x)=\operatorname{sinc}(x)= \begin{cases}\frac{\sin (\pi x)}{\pi x} & \text { if } x \neq 0, \\ 1 & \text { if } x=0,\end{cases}
$$

is the basic (Shannon) scaling function.

\subsection{Recovery of densities $f_{d}(\cdot)$ and $f_{f}(\cdot)$}

We collectively denote $f_{d}(\cdot)$ and $f_{f}(\cdot)$ by $f_{s}, s \in\{d, f\}$. Following the wavelets theory in Section 4.1

$$
f_{s}(t) \approx \mathcal{P}_{m_{s}} f(t)=\sum_{k_{s} \in \mathbb{Z}} c_{m_{s}, k_{s}}^{s} \varphi_{m_{s}, k_{s}}(t)
$$

Since the function $f_{s}$ is supported on the finite interval $\left[a_{s}, b_{s}\right]=[0, T], s \in\{d, f\}$, without loss of density mass, we have the following approximation

$$
\mathcal{P}_{m_{s}} f(t) \approx f_{s, m_{s}}(t)=\sum_{k_{s}=\left\lfloor 2^{m_{s}} a_{s}\right\rfloor}^{\left\lceil 2^{m_{s}} b_{s}\right\rceil} c_{m_{s}, k_{s}}^{s} \varphi_{m_{s}, k_{s}}(t) \equiv \sum_{k_{s}=0}^{\left\lceil 2^{m_{s}} T\right\rceil} c_{m_{s}, k_{s}}^{s} \varphi_{m_{s}, k_{s}}(t),
$$

where $\lfloor x\rfloor$ denotes the greatest integer less than or equal to $x$, and $\lceil x\rceil$ denotes the smallest integer greater than or equal to $x$. This function could be further approximated by

$$
f_{s, m_{s}}(t) \approx f_{s, m_{s}}^{*}(t)=\sum_{k_{s}=\left\lfloor 2^{m_{s}} a_{s}\right\rfloor}^{\left\lceil 2^{m_{s}} b_{s}\right\rceil} c_{m_{s}, k_{s}}^{s, *} \varphi_{m_{s}, k_{s}}(t)
$$

due to the approximation (see the details in Ortiz-Gracia and Oosterlee (2016))

$$
c_{m_{s}, k_{s}}^{s} \approx c_{m_{s}, k_{s}}^{s, *}=\frac{2^{m_{s} / 2}}{2^{J_{s}-1}} \sum_{j_{s}=1}^{2^{J_{s}-1}} \Re\left\{\Psi_{s}\left(\frac{\left(2 j_{s}-1\right) \pi 2^{m_{s}}}{2^{J_{s}}}\right) e^{-\frac{i k_{s} \pi\left(2 j_{s}-1\right)}{2_{s}}}\right\},
$$

where $J_{s}, s \in\{d, f\}$, is the truncation parameter. Substituting (4.6) into (3.7) gives

$$
\begin{aligned}
v(0, z)=2 \pi \sum_{k_{d}=\left\lfloor 2^{m_{d}} a_{d}\right\rfloor}^{\left\lceil 2^{\left.m_{d} b_{d}\right\rceil}\right.} \quad \sum_{k_{f}=\left\lfloor 2^{m_{f}} a_{f}\right\rfloor}^{\left\lceil 2^{m_{f}} b_{f}\right\rceil} c_{m_{d}, k_{d}}^{d, *} c_{m_{f}, k_{f}}^{f, *} \sum_{n=0}^{\infty} \frac{(\lambda T)^{n}}{n !} e^{-\lambda T} \\
\mathbb{E}^{\mathbb{Q}}\left[\phi *\left(t \mapsto \frac{e^{-\frac{(t+F+n \tilde{\mu})^{2}}{2\left(2 G+n \tilde{\sigma}^{2}\right)}}}{\sqrt{2 G+n \tilde{\sigma}^{2}}}\right) *\left(t \mapsto e^{t} \varphi_{m_{d}, k_{d}}(-t)\right) *\left(\varphi_{m_{f}, k_{f}}\right)(z)\right] .
\end{aligned}
$$


296 Next, we focus on $\left(t \mapsto \frac{e^{-\frac{(t+F+n \tilde{\mu})^{2}}{2\left(2 G+n \tilde{\sigma}^{2}\right)}}}{\sqrt{2 G+n \tilde{\sigma}^{2}}}\right) *\left(t \mapsto e^{t} \varphi_{m_{d}, k_{d}}(-t)\right)$. Using the fact that $\varphi$ is even, we have

297

$$
\left(t \mapsto \frac{e^{-\frac{1}{2} \frac{(t+F+n \tilde{\mu})^{2}}{2\left(2 G+n \tilde{\sigma}^{2}\right)}}}{\sqrt{2\left(2 G+n \tilde{\sigma}^{2}\right)}}\right) *\left(t \mapsto e^{t} \varphi_{m_{d}, k_{d}}(-t)\right)(x)=\frac{2^{m_{d} / 2}}{\sqrt{2 \pi}} \int_{\mathbb{R}} \frac{e^{-\frac{1}{2} \frac{(x-t+F+n \tilde{\mu})^{2}}{2\left(2 G+n \tilde{\sigma}^{2}\right)}}}{\sqrt{2\left(2 G+n \tilde{\sigma}^{2}\right)}} \operatorname{sinc}\left(2^{m_{d}} t+k_{d}\right) \mathrm{d} t
$$

It turns out that, thanks to certain local approximation properties of wavelets, the expression (4.9) can be further simplified to a single integral by using a highly accurate approximation for the integral term. To this end, we recall the following theorem in Stenger (2011)

Theorem 4.1 (Theorem 1.3.2 of Stenger (2011)). Let $f$ be defined on $\mathbb{R}$, and let its Fourier transform, denoted by $\hat{f}$, be such that, for some positive constant $d$

$$
|\hat{f}(\xi)|=\mathcal{O}\left(e^{-d|\xi|}\right), \quad \xi \rightarrow \pm \infty .
$$

Then, as $a \rightarrow 0$,

$$
\frac{1}{a} \int_{\mathbb{R}} f(y) \mathcal{S}(k, a)(y) \mathrm{d} y-f(k a)=\mathcal{O}\left(e^{-\frac{\pi d}{a}}\right),
$$

where $\mathcal{S}(k, a)(y):=\operatorname{sinc}\left(\frac{y}{a}-k\right)$.

To apply this theorem to function $f(t)=\frac{e^{-\frac{(x-t+F+n \tilde{\mu})^{2}}{2\left(2 G+n \tilde{\sigma}^{2}\right)}}}{\sqrt{2 G+n \tilde{\sigma}^{2}}}$, we need to check whether its Fourier transform satisfies the condition (4.10). Simple algebra shows that

$$
\mathcal{F} f(\xi)=\frac{1}{\sqrt{2 \pi}} \int_{\mathbb{R}} \frac{e^{-\frac{(x-t+F+n \tilde{\tilde{\mu}})^{2}}{2\left(2 G+n \tilde{\sigma}^{2}\right)}+t}}{\sqrt{2 G+n \tilde{\sigma}^{2}}} e^{-i \xi t} \mathrm{~d} t=e^{x+F+n \tilde{\mu}+\left(G+\frac{1}{2} n \tilde{\sigma}^{2}\right)\left(1-\xi^{2}\right)-i \xi\left(x+F+n \tilde{\mu}+2 G+n \tilde{\sigma}^{2}\right)} .
$$

Now, we notice that coefficient $G$ in the quadratic term in the exponent of that term is strictly positive (see (3.4)). In addition, $G$ and $F$ are a also bounded, due to the boundedness of the variance process (Andersen and Piterbarg, 2007). It follows that, for a given $n$, the Fourier transform of $f(\cdot)$ satisfies the hypothesis of Theorem 4.1. Hence, we can apply Theorem 4.1 with $a=2^{-m_{d}}$ and $k=-k_{d}$. We obtain the following approximation

$$
\left(t \mapsto \frac{e^{-\frac{(t+F+n \tilde{\mu})^{2}}{2\left(2 G+n \tilde{\sigma}^{2}\right)}}}{\sqrt{2 G+n \tilde{\sigma}^{2}}}\right) *\left(t \mapsto e^{t} \varphi_{m_{d}, k_{d}}(-t)\right)(x) \approx \frac{1}{\sqrt{2 \pi} 2^{m_{d} / 2}} f\left(-\frac{k_{d}}{2^{m_{d}}}\right)=\frac{e^{-\frac{\left(x+F+n \tilde{\mu}+k_{d} / 2^{\left.m_{d}\right)^{2}}\right.}{2\left(2 G+n \tilde{\sigma}^{2}\right)}-k_{d} / 2^{m_{d}}}}{\sqrt{2 \pi} 2^{m_{d} / 2 \sqrt{2 G+n \tilde{\sigma}^{2}}}} .
$$

With (4.11), the quantity inside the expectation of (4.8) becomes

$$
\frac{e^{-k_{d} / 2^{m_{d}}}}{\sqrt{2 \pi} 2^{m_{d} / 2}} \mathbb{E}^{\mathbb{Q}}\left[\phi *\left(t \mapsto \frac{e^{-\frac{\left(t+F+n \tilde{\mu}+k_{d} / 2^{m_{d}}\right)^{2}}{2\left(2 G+n \tilde{\sigma}^{2}\right)}}}{\sqrt{2 G+n \tilde{\sigma}^{2}}}\right) *\left(\varphi_{m_{f}, k_{f}}\right)(z)\right] .
$$

We repeat the same process for the convolution product with the Shannon expansion of the density of the time-integrated foreign interest rate process, and obtain

$$
\left(t \mapsto \frac{e^{-\frac{\left(t+F+n \tilde{\mu}+k_{d} / 2^{m} d\right)^{2}}{2\left(2 G+n \tilde{\sigma}^{2}\right)}}}{\sqrt{2 G+n \tilde{\sigma}^{2}}}\right) * \varphi_{m_{f}, k_{f}}(x)=\frac{2^{m_{f} / 2}}{\sqrt{2 \pi}} \int_{\mathbb{R}} \frac{e^{-\frac{\left(x-t+F+n \tilde{\mu}+k_{d} / 2^{m_{d}}\right)^{2}}{2\left(2 G+n \tilde{\sigma}^{2}\right)}}}{\sqrt{2 G+n \tilde{\sigma}^{2}}} \varphi\left(2^{m_{f}} t-k_{f}\right) \mathrm{d} t
$$

321 Using again Theorem 4.1 with $a=2^{-m_{f}}, k=k_{f}$, and $f(t)=\frac{e^{-\frac{\left(x-t+F+n \tilde{\mu}+k_{d} / 2^{m} d\right)^{2}}{2\left(2 G+n \tilde{\sigma}^{2}\right)}}}{\sqrt{2 G+n \tilde{\sigma}^{2}}}$, we obtain the 
following approximation

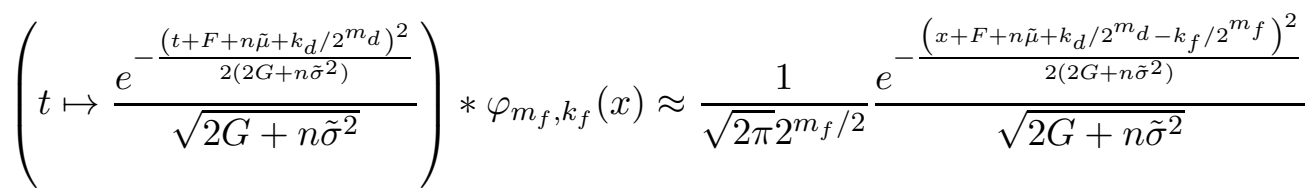

Putting everything together into (4.8), and by letting $M=m_{d}+m_{f}$, and

$$
F_{n}=F+n \tilde{\mu}, \quad G_{n}=G+\frac{n \tilde{\sigma}^{2}}{2},
$$

where $F$ and $G$ are given in (3.4), we obtain the pricing formula under the "two-density" treatment

$$
\begin{aligned}
& v(0, z)=\sum_{k_{d}=\left\lfloor 2^{m_{d}} a_{d}\right\rfloor}^{\left\lceil 2^{\left.m_{d} b_{d}\right\rceil}\right.} \sum_{k_{f}=\left\lfloor 2^{m_{f}} a_{f}\right\rfloor}^{\left\lceil 2^{m_{f}} b_{f}\right\rceil} \frac{e^{-k_{d} / 2^{m_{d}}}}{2^{M / 2}} \quad c_{m_{d}, k_{d}}^{d, *} c_{m_{f}, k_{f}}^{f, *} \sum_{n=0}^{\infty} \frac{(\lambda T)^{n}}{n !} e^{-\lambda T} \\
& \mathbb{E}^{\mathbb{Q}}\left[\phi *\left(t \mapsto \frac{e^{-\frac{\left(t+F_{n}+\frac{k_{d}}{2^{m} d}-\frac{k_{f}}{2^{m} f}\right)^{2}}{2\left(2 G_{n}\right)}}}{\sqrt{2 G_{n}}}\right)(z)\right] .
\end{aligned}
$$

Here, $a_{s}=0, b_{s}=T$, and $c_{m_{s}, k_{s}}^{s, *}, s=\{d, f\}$, are given in (7.2).

\subsection{Recovery of $f_{c}(\cdot)$}

Since $f_{s}(\cdot), s=\{d, f\}$, is supported on $\left[a_{s}, b_{s}\right]$, the support of $f_{c}(\cdot)$ is contained within

$$
\left[a_{c}, b_{c}\right]=\left[a_{d}-b_{f}, b_{d}-a_{f}\right]
$$

As indicated in the previous section, $a_{d}=a_{f}=0$ and $b_{d}=b_{f}=T$, we have that $a_{c}=-T$ and $b_{c}=T$. Following the same steps as in the previous sections gives

$$
f_{c, m_{c}}(t) \approx f_{c, m_{c}}^{*}(t)=\sum_{k_{c}=\left\lfloor 2^{m_{c}} a_{c}\right\rfloor}^{\left\lceil 2^{m_{c}} b_{c}\right\rceil} c_{m_{c}, k_{c}}^{*} \varphi_{m_{c}, k_{c}}(t),
$$

where

$$
c_{m_{c}, k_{c}}^{*}=\frac{2^{m_{c} / 2}}{2^{J_{c}-1}} \sum_{j=1}^{2^{J_{c}-1}} \Re\left\{\Psi_{c}\left(\frac{(2 j-1) \pi 2^{m_{c}}}{2^{J_{c}}}\right) e^{-\frac{i k_{c} \pi(2 j-1)}{2^{J_{c}}}}\right\},
$$

from which, we obtain the following pricing formula under the "combined-density" treatment:

$$
v(0, z)=\sum_{k_{c}=\left\lfloor 2^{m_{c}} a_{c}\right\rfloor}^{\left\lceil 2^{\left.m_{c} b_{c}\right\rceil}\right.} \frac{1}{2^{m_{c} / 2}} c_{m_{c}, k_{c}}^{*} \sum_{n=0}^{\infty} \frac{(\lambda T)^{n}}{n !} e^{-\lambda T} \mathbb{E}^{\mathbb{Q}}\left[\phi *\left(t \mapsto \frac{e^{-\frac{\left(t+F_{n}+\frac{k_{c}}{2 m_{c}}\right)^{2}}{2\left(2 G_{n}\right)}}}{\sqrt{2 G_{n}}}\right)(z)\right]
$$

Remark 4.1. We note that the "two-density" treatment involves recovering, using Shannon wavelets, two different densities. This results in a double summation for the coefficients of the two interest rates in the pricing formula (4.13). On the other hand, the "combined-density" treatment results in a pricing formula that involves only one summation for the coefficients of both interest rates, see (4.17). As a result, it is expected that the "combined-density" treatment is more efficient than the "two-density" treatment. We will demonstrate this through numerical experiments in Section 8. 


\section{Application to plain-vanilla European options}

To illustrate the method, we consider a standard call and a put option with the payoff functions

$$
\phi(z)= \begin{cases}e^{z}-K & \text { call option } \\ K-e^{z} & \text { put option }\end{cases}
$$

where $K$ is the strike price. We have the following results.

Theorem 5.1 ("two-density" treatment). Under model (2.1) and with the "two-density" treatment, the prices of plain-vanilla European call and put options are respectively given by

352

3

54

55

$$
\begin{aligned}
V^{\text {call }}(0, S(0)) \approx & \frac{1}{2^{M / 2}} \sum_{k_{d}=\left\lfloor 2^{m_{d}} a_{d}\right\rfloor}^{\left\lceil 2^{\left.m_{d} b_{d}\right\rceil}\right.} \sum_{k_{f}=\left\lfloor 2^{m_{f}} a_{f}\right\rfloor}^{\left\lceil 2^{\left.m_{f} b_{f}\right\rceil}\right.} c_{m_{d}, k_{d}}^{d, *} c_{m_{f}, k_{f}}^{f, *} \\
& \sum_{n=0}^{\infty} \frac{(\lambda T)^{n}}{n !} e^{-\lambda T} \mathbb{E}^{\mathbb{Q}}\left[S(0) e^{F_{n}+G_{n}-\frac{k_{f}}{2^{m_{f}}}} \mathcal{N}\left(d_{+, n}\right)-K e^{-\frac{k_{d}}{2^{m_{d}}}} \mathcal{N}\left(d_{-, n}\right)\right] \\
V^{\text {put }}(0, S(0)) \approx \quad & \frac{1}{2^{M / 2}} \sum_{k_{d}=\left\lfloor 2^{m_{d}} a_{d}\right\rfloor}^{\left\lceil 2^{m_{d}}\right\rfloor} \sum_{k_{f}=\left\lfloor 2^{m_{f}} a_{f}\right\rfloor}^{\left\lceil 2^{m_{f}} b_{f}\right\rceil} c_{m_{d}, k_{d}}^{d, *} c_{m_{f}, k_{f}}^{f, *} \\
& \sum_{n=0}^{\infty} \frac{(\lambda T)^{n}}{n !} e^{-\lambda T} \mathbb{E}^{\mathbb{Q}}\left[K e^{-\frac{k_{d}}{2^{m} d}} \mathcal{N}\left(-d_{-, n}\right)-S(0) e^{F_{n}+G_{n}-\frac{k_{f}}{2^{m} f}} \mathcal{N}\left(-d_{+, n}\right)\right],
\end{aligned}
$$

where $a_{s}=0, b_{s}=T$, with $s=\{d, f\}, M=m_{d}+m_{f}$, and

$$
d_{+, n}=\frac{\log \left(\frac{S(0)}{K}\right)+\frac{k_{d}}{2^{m} d}-\left(\frac{k_{f}}{2^{m_{f}}}-F_{n}-G_{n}\right)}{\sqrt{2 G_{n}}}+\sqrt{2 G_{n}}, \quad d_{-, n}=d_{+, n}-\sqrt{2 G_{n}},
$$

and $F_{n}$ and $G_{n}$ are given in (4.12).

Proof. For a call option, $\phi(t)=\left(e^{t}-K\right)^{+}$. Thus, noting (4.13) and by convolution theorem, we have

$\phi *\left(t \mapsto \frac{1}{\sqrt{2 G_{n}}} e^{-\frac{1}{2\left(2 G_{n}\right)}\left(t+F_{n}+\frac{k_{d}}{2^{m} d}-\frac{k_{f}}{2^{m} f}\right)^{2}}\right)=\frac{1}{\sqrt{4 \pi G_{n}}} \int_{\log (K)}^{+\infty}\left(e^{t}-K\right) e^{-\frac{1}{2\left(2 G_{n}\right)}\left(x-t+F_{n}+\frac{k_{d}}{2^{m} d}-\frac{k_{f}}{2^{m} f}\right)^{2}} \mathrm{~d} t$

The change of variable $u=\frac{t-x-F_{n}-\frac{k_{d}}{2^{m^{m}}}+\frac{k_{f}}{2^{m f} f}}{\sqrt{2 G_{n}}}$, noting the definition of $d_{+, n}$ and $d_{-, n}$ in (5.2), together with some algebra, yields

$$
\begin{aligned}
& \phi *\left(t \mapsto \frac{1}{\sqrt{2 G_{n}}} e^{-\frac{1}{2\left(2 G_{n}\right)}\left(t+F_{n}+\frac{k_{d}}{2^{m} d}-\frac{k_{f}}{2^{m} f}\right)^{2}}\right)=\frac{1}{\sqrt{2 \pi}} \int_{-d_{-, n}}^{+\infty}\left(e^{\sqrt{2 G_{n}} u+x+F_{n}+\frac{k_{d}}{2^{m^{\prime}} d}-\frac{k_{f}}{2^{m_{f}}}}-K\right) e^{-\frac{u^{2}}{2}} \mathrm{~d} u \\
& =\frac{1}{\sqrt{2 \pi}}\left(e^{x+F_{n}+G_{n}+\frac{k_{d}}{2^{m^{\prime}} d}-\frac{k_{f}}{2^{m_{f}}}} \int_{-\infty}^{d_{+, n}} e^{-\frac{v^{2}}{2}} \mathrm{~d} v-K \int_{-\infty}^{d_{-, n}} e^{-\frac{u^{2}}{2}} \mathrm{~d} u\right) .
\end{aligned}
$$

Substituting this into (4.13) with further algebra yields $V^{\text {call }}(0, S(0))$ in (5.1). For a put option, $\phi(t)=\left(K-e^{t}\right)^{+}$, and performing similar integration steps yields the desired result.

For the "combined-density" treatment, the results are given in the following theorem, which can be proved following the same steps as those in the proof for Theorem 5.1 . 
Theorem 5.2 ("combined-density"treatment). Under model (2.1) and with the "combined-density" treatment, the prices of plain-vanilla European call and put options are respectively given by

$$
\begin{array}{rll}
V^{\text {call }}(0, S(0)) \approx \frac{1}{2^{m_{c} / 2}} \sum_{k_{c}=\left\lfloor 2^{m_{c}} a_{c}\right\rfloor}^{\left\lceil 2^{\left.m_{c} b_{c}\right\rceil} c_{m_{c}, k_{c}}\right.} & \sum_{n=0}^{\infty} \frac{(\lambda T)^{n}}{n !} e^{-\lambda T} \\
& \mathbb{E}^{\mathbb{Q}}\left[S(0) e^{F_{n}+G_{n}+\frac{k_{c}}{2^{m_{c}}}} \mathcal{N}\left(\hat{d}_{+, n}\right)-K \mathcal{N}\left(\hat{d}_{-, n}\right)\right] \\
V^{\text {put }}(0, S(0)) \approx \frac{1}{2^{m_{c} / 2}} \sum_{k_{c}=\left\lfloor 2^{m_{c}} a_{c}\right\rfloor}^{\left\lceil 2^{\left.m_{c} b_{c}\right\rceil}\right.} c_{m_{c}, k_{c}}^{*} & \sum_{n=0}^{\infty} \frac{(\lambda T)^{n}}{n !} e^{-\lambda T} \\
& \mathbb{E}^{\mathbb{Q}}\left[K \mathcal{N}\left(-\hat{d}_{-, n}\right)-S(0) e^{F_{n}+G_{n}+\frac{k_{c}}{2^{m_{c}}}} \mathcal{N}\left(-\hat{d}_{+, n}\right)\right],
\end{array}
$$

where $a_{c}=-T, b_{c}=T$, and

$$
\hat{d}_{+, n}=\frac{\log \left(\frac{S(0)}{K}\right)+F_{n}+\frac{k_{c}}{2^{m_{c}}}}{\sqrt{2 G_{n}}}+\sqrt{2 G_{n}}, \quad \hat{d}_{-, n}=\hat{d}_{+, n}-\sqrt{2 G_{n}},
$$

and $F_{n}$ and $G_{n}$ are given in (4.12).

We now make a few interesting observations about the quantity inside the expectation $\mathbb{E}^{\mathbb{Q}}(\cdot)$ in the formulas in Theorem [5.1. This quantity exactly resembles the closed-form solution of foreign exchange call/put options under the Garman-Kohlhagen model (Garman and Kohlhagen, 1983) in which the interest rates and the variance are assumed to be constant. In particular, this quantity can be obtained by substituting into the closed-form formulas of Garman and Kohlhagen (1983) the (conditionally) constant domestic and foreign interest rates $\frac{\frac{k_{d}}{2^{m_{d}}}}{T}$ and $\frac{\frac{k_{f}}{2^{m_{f}}}-F_{n}-G_{n}}{T}$, respectively, and the (conditionally) constant variance $\frac{2 G_{n}}{T}$. We note that these domestic and foreign interest rates, as well as the variance, are conditional on the $\nu$ path and on having $n$-jumps in the foreign exchange rate $S$ during the life of the option, and hence are (conditionally) constant. In some sense, the quantity $\frac{\frac{k_{d}}{2^{m} d}}{T}$ can be viewed as the contribution of the $k_{d}$-th wavelet in the wavelet decomposition of the "effective average" domestic interest rate, namely $\frac{\int_{0}^{T} r_{d}(t) \mathrm{d} t}{T}$. The quantity $\frac{\frac{k_{f}}{2^{m} f}-F_{n}-G_{n}}{T}$ can also be viewed as containing a component representing the contribution of the $k_{f}$-th wavelet with respect to the decomposition of $\frac{\int_{0}^{T} r_{f}(t) \mathrm{d} t}{T}$, and another component due to presence of jumps in $S$. With respect to the "combined-density" treatment (Theorem [5.2), one can obtain the formulas of the quantity inside the expectation by substituting into the Garman-Kohlhagen formulas the constant domestic rate equal to zero, the (conditionally) constant foreign interest rate equal to $\frac{-\frac{k_{c}}{2^{m_{c}}}-F_{n}-G_{n}}{T}$, and the (conditionally) constant variance equal to $\frac{2 G_{n}}{T}$.

\section{Efficient computation of $\mathbb{E}^{\mathbb{Q}}[\cdot]$ via Shannon wavelets.}

The focus of this section is efficient computation of the expectation $\mathbb{E}^{\mathbb{Q}}[\cdot]$ in the formulas (55.1)-(5.3) presented in Theorems 5.1 and 5.2 by a Shannon wavelets method. 


\subsection{Recovery of $\int_{0}^{T} \nu(t) \mathrm{d} t \mid \nu(T)$}

Examination of (3.4) shows that $G$ depends only on $\int_{0}^{T} \nu(t) \mathrm{d} t$, while $F$ depends on both $\int_{0}^{T} \nu(t) \mathrm{d} t$ and $\int_{0}^{T} \nu(t) \mathrm{d} W_{\nu}(t)$. From (2.4) $)$, we note that

$$
\int_{0}^{T} \sqrt{\nu(t)} \mathrm{d} W_{\nu}(t)=\frac{\nu(T)-\nu_{0}-\kappa_{\nu} \bar{\nu} T+\kappa_{\nu} \int_{0}^{T} \nu(t) \mathrm{d} t}{\sigma_{\nu}} .
$$

Therefore, $F$ can be expressed in terms of $\int_{0}^{T} \nu(t) \mathrm{d} t$ and the terminal value $\nu(T)$ of the variance. For presentation purposes, we write the formulas in (5.1) and (5.3) in the following generic form:

$$
V(0, S(0))=\sum_{\ell \in \mathcal{L}} c_{\ell} \sum_{n \in \mathbb{N}} d_{n} \mathbb{E}^{\mathbb{Q}}\left[g_{\ell, n}\left(\int_{0}^{T} \nu(t) \mathrm{d} t, \nu(t)\right)\right] .
$$

Here, $\mathcal{L}$ is a finite set, $\left\{c_{\ell}\right\}_{\ell \in \mathcal{L}}$, and $\left\{d_{n}\right\}_{n \in \mathbb{N}}$, are real constants and $\left\{g_{\ell, n}\right\}_{(\ell, n) \in \mathcal{L} \times \mathbb{N}}$, are real functions given by the quantity inside the expectation $\mathbb{E}^{\mathbb{Q}}[\cdot]$ in formulas (5.1)-(5.3). By conditioning on $\nu(T)$, we have

$$
\mathbb{E}^{\mathbb{Q}}\left[g_{\ell, n}\left(\int_{0}^{T} \nu(t) \mathrm{d} t, \nu(T)\right)\right]=\mathbb{E}^{\mathbb{Q}}\left[\mathbb{E}^{\mathbb{Q}}\left[g_{\ell, n}\left(\int_{0}^{T} \nu(t) \mathrm{d} t, \nu(t)\right) \mid \nu(T)\right]\right] .
$$

This form allows us to take advantage of the known characteristic function of the time-integrated CIR process conditional on the terminal value.

Let $f(\cdot \mid y)$ the density of the time-integrated variance process conditional on the terminal value $\nu(T)=y$, where $y \in\left[0, y_{0}\right]$ for a $y_{0}>0$. We can assume that $f(\cdot \mid y)$ is supported on the interval $[0, T]$. From (6.1) and (6.2), the option can be represented by

$$
V(0, S(0))=\sum_{\ell \in \mathcal{L}} c_{\ell} \sum_{n \in \mathbb{N}} d_{n} \int_{0}^{y_{0}}\left[\int_{0}^{T} g_{\ell, n}(x, y) f(x \mid y) \mathrm{d} x\right] w(y) \mathrm{d} y .
$$

Here, $w(\cdot)$ is the density of the terminal value of the CIR process given by (Cox et al., 1985a)

$$
w(y):=\zeta e^{-\zeta\left(\nu(0) e^{-\kappa_{\nu} T}+y\right)} \cdot\left(\frac{y}{\nu(0) e^{-\kappa_{\nu} T}}\right)^{\frac{q}{2}} \cdot I_{q}\left(2 \zeta e^{-\frac{1}{2} \kappa_{\nu} T} \sqrt{\nu(0) y}\right),
$$

where $q:=\frac{2 \kappa_{\nu} \bar{\nu}}{\sigma_{\nu}^{2}}-1, \zeta:=\frac{2 \kappa_{\nu}}{\left(1-e^{-\kappa_{\nu} T}\right) \sigma_{\nu}^{2}}$ and $I_{q}(x)$ is the modified Bessel function of the first kind with order $q$.

To evaluate the integral (6.3), the conditional density $f(\cdot \mid y), y \in\left[0, y_{0}\right]$, first needs to be approximated, since it is not known in closed-form. Following the same methodology as in Section 4, noting that the function $f(\cdot \mid y)$ is supported on the interval $[0, T]$, we can approximate this function by its Shannon wavelets expansion as follows

$$
f^{*}(x \mid y) \approx \sum_{k_{\nu}=0}^{\left\lceil 2^{m_{\nu}} T\right\rceil} c_{m_{\nu}, k_{\nu}}^{\nu, *}(y) \varphi_{m_{\nu}, k_{\nu}}(x)
$$

where $c_{m_{\nu}, k_{\nu}}^{\nu, *}$ are given by

$$
c_{m_{\nu}, k_{\nu}}^{\nu, *}=\frac{2^{m_{\nu} / 2}}{2^{J_{\nu}-1}} \sum_{j=1}^{2^{J_{\nu}-1}} \Re\left\{\Psi^{C}\left(\frac{(2 j-1) \pi 2^{m_{\nu}}}{2^{J_{\nu}}} \mid y\right) e^{-i \frac{k_{\nu} \pi(2 j-1)}{2^{J} \nu}}\right\} .
$$


Here, $\Psi^{C}(\xi \mid \nu(T))$ is known in closed-form (Broadie and Kaya, 2006)

$$
\begin{aligned}
\Psi^{C}(\xi \mid y)= & \frac{I_{q}\left(\sqrt{\nu(T) \nu(0)} \frac{4 \gamma(\xi) e^{-\frac{1}{2} \gamma(\xi) T}}{\sigma_{\nu}^{2}\left(1-e^{-\gamma(\xi) T}\right)}\right)}{I_{q}\left(\sqrt{\nu(T) \nu(0)} \frac{4 \kappa_{\nu} e^{-\frac{1}{2} \kappa_{\nu} T}}{\sigma_{\nu}^{2}\left(1-e^{-\kappa_{\nu} T}\right)}\right)} \times \frac{\gamma(\xi) e^{-\frac{1}{2}\left(\gamma(\xi)-\kappa_{\nu}\right) T}\left(1-e^{-\kappa_{\nu} T}\right)}{\kappa_{\nu}\left(1-e^{-\gamma(\xi) T}\right)} \\
& \times \exp \left(\frac{\nu(0)+\nu(T)}{\sigma_{\nu}^{2}}\left[\frac{\kappa_{\nu}\left(1+e^{-\kappa_{\nu} T}\right)}{1-e^{-\kappa_{\nu} T}}-\frac{\gamma(\xi)\left(1+e^{-\gamma(\xi) T}\right)}{1-e^{-\gamma(\xi) T}}\right]\right), .
\end{aligned}
$$

with $\gamma(\xi):=\sqrt{\kappa_{\nu}^{2}-2 i \sigma_{\nu}^{2} \xi}$. We note that, if a time-dependent correlation function $\rho_{t}$ were used, we would need to know the characteristic function of $\int_{0}^{T} \rho_{t} \nu_{t} d W_{t}$ conditional on $\nu_{T}$, which does not appear to be readily available for a general $\rho_{t}$.

\subsection{Approximation formulas to $V(0, S(0))$}

Following the same methodology as in Dang and Ortiz-Gracia (2018), for a fixed level of resolution $m_{\nu}$ and a fixed truncation parameter $J_{\nu}$, replacing the conditional density function $f(\cdot \mid y)$ in (6.3) by the finite approximation (6.5) gives us the approximation $V_{1}(0, S(0))$ to the option price $V(0, S(0))$

$$
V(0, S(0)) \approx V_{1}(0, S(0))=\sum_{\ell \in \mathcal{L}} c_{\ell} \sum_{n \in \mathbb{N}} d_{n} \int_{0}^{y_{0}}\left[\sum_{k_{\nu}=0}^{\left[2^{m_{\nu}} T\right\rceil} c_{m_{\nu}, k_{\nu}}^{\nu, *}(y) \int_{0}^{T} g_{\ell, n}(x, y) \varphi_{m_{\nu}, k_{\nu}}(x) \mathrm{d} x\right] w(y) \mathrm{d} y
$$

Applying Theorem 4.1 with $a=\frac{1}{2^{m_{\nu} / 2}}$ to function $g_{\ell, n}(\cdot, \cdot)$ in the above integral gives

$$
\int_{0}^{T} g_{\ell, n}(x, y) \phi_{m_{\nu}, k_{\nu}}(x) \mathrm{d} x \approx \frac{1}{2^{m_{\nu} / 2}} g_{\ell, n}\left(\frac{k_{\nu}}{2^{m_{\nu}}}, y\right)
$$

Thus, we arrive at the approximation $V_{2}(0, S(0))$ of $V_{1}(0, S(0))$

$$
V_{1}(0, S(0)) \approx V_{2}(0, S(0))=\frac{1}{2^{m_{\nu} / 2}} \sum_{\ell \in \mathcal{L}} c_{\ell} \sum_{n \in \mathbb{N}} d_{n} \int_{0}^{y_{0}}\left[\sum_{k_{\nu}=0}^{\left\lceil 2^{m_{\nu}} T\right\rceil} c_{m_{\nu}, k_{\nu}}(y) g_{\ell, n}\left(\frac{k_{\nu}}{2^{m_{\nu}}}, y\right)\right] w(y) \mathrm{d} y,(6.8)
$$

where $c_{m_{\nu}, k_{\nu}}^{\nu, *}(y)$ are defined in (6.6). Finally, the integral in (6.8) can be approximated by means of the composite trapezoidal rule.

When the Feller condition for the variance process is not satisfied, i.e. $2 \kappa_{\nu} \bar{\nu}<\sigma_{\nu}^{2}$, which is common in practice, the accuracy of the composite trapezoidal rule applied to (6.8) may be affected. Following Fang and Oosterlee (2011), we use the change of variable $v=\ln (y)$ in (6.8), and this gives

$$
V_{2}(S(0), 0, \cdot)=\frac{1}{2^{m_{\nu} / 2}} \sum_{\ell \in \mathcal{L}} c_{\ell} \sum_{n \in \mathbb{N}} d_{n} \int_{-\infty}^{\ln \left(y_{0}\right)}\left[\sum_{k_{\nu}=0}^{\left[2^{m_{\nu}} T\right\rceil} c_{m_{\nu}, k_{\nu}}^{\nu, *}\left(e^{v}\right) g_{\ell, n}\left(\frac{k_{\nu}}{2^{m_{\nu}}}, e^{v}\right)\right] \bar{w}(v) \mathrm{d} v,
$$

where

$$
\bar{w}(v)=e^{v} \tilde{w}(v), \text { with } \quad \tilde{w}(v):=\zeta e^{-\zeta\left(\nu(0) e^{-\kappa_{\nu} T}+e^{v}\right)} \cdot\left(\frac{e^{v}}{\nu(0) e^{-\kappa_{\nu} T}}\right)^{\frac{q}{2}} \cdot I_{q}\left(2 \zeta e^{-\frac{1}{2} \kappa_{\nu} T} \sqrt{\nu(0) e^{v}}\right) .
$$

\subsection{Implementation}

We first briefly describe an iterative procedure to determine an appropriate truncated integration domain, denoted by $\left[a_{v}, b_{v}\right]$, for the log-variance density $\bar{w}(v)$, according to a pre-defined tolerance $\epsilon_{\text {tol }}$. We denote by $\left[a_{v}^{(j)}, b_{v}^{(j)}\right], j=0,1, \ldots$, the interval at the $j$-th iteration. Given an initial guess $\left[a_{v}^{(0)}, b_{v}^{(0)}\right]$, we iteratively modify the interval until the condition $\bar{w}(v)<\epsilon_{\text {tol }}$ for $v \in \mathcal{D}$ is met, where 
$\mathcal{D}=\left(-\infty, a_{v}^{(j)}\right) \cup\left(b_{v}^{(j)}, \ln \left(y_{0}\right)\right)$, for some $j$, after which the truncated integration domain is taken to be $\left[a_{v}^{(j)}, b_{v}^{(j)}\right]$.

Using a first-order Taylor expansion of $\ln (\nu(T))$, we have the approximations

$$
\mathbb{E}[\ln (\nu(T))] \approx \ln (\mathbb{E}[\nu(T)]), \quad \mathbb{V}[\ln (\nu(T))] \approx \frac{\mathbb{V}[\nu(T)]}{\mathbb{E}[\nu(T)]^{2}}
$$

Then, taking into account that the left tail of the density of the log-variance density $\bar{w}(v)$ decays slower than the right tail, we consider the following initial interval $\bar{w}(v)$

$$
\left[a_{v}^{(0)}, b_{v}^{(0)}\right]=\left[\ln (\mathbb{E}[\nu(T)])-7 \frac{\mathbb{V}[\nu(T)]}{\mathbb{E}[\nu(T)]^{2}}, \ln (\mathbb{E}[\nu(T)])+3 \frac{\mathbb{V}[\nu(T)]}{\mathbb{E}[\nu(T)]^{2}}\right],
$$

where, as given in Cox et al. (1985b),

$$
\begin{aligned}
& \mathbb{E}[\nu(T)]=\nu(0) e^{-\kappa_{\nu} T}+\bar{\nu}\left(1-e^{-\kappa_{\nu} T}\right) \\
& \mathbb{V}[\nu(T)]=\nu(0) \frac{\sigma_{\nu}^{2}}{\kappa_{\nu}} e^{-\kappa_{\nu} T}-e^{-2 \kappa_{\nu} T}+\bar{\nu} \frac{\sigma_{\nu}^{2}}{2 \kappa_{\nu}}\left(1-e^{-\kappa_{\nu} T}\right)^{2} .
\end{aligned}
$$

Now, given $\left[a_{v}^{(0)}, b_{v}^{(0)}\right]$, we propose two methods for finding the final interval $\left[a_{v}^{(j)}, b_{v}^{(j)}\right]$. The first one involves the Newton iteration, for which we need the derivative of $\tilde{w}(v)$

$$
\tilde{w}^{\prime}(v):=\zeta e^{-u-\zeta e^{v}+v}\left(\frac{\zeta e^{v}}{u}\right)^{\frac{q}{2}} \cdot\left[\left(-\zeta e^{v}+q+1\right) \cdot I_{q}\left(2 \sqrt{\zeta e^{v} u}\right)+\zeta \sqrt{\nu(0) e^{v-\kappa_{\nu} T}} \cdot I_{q+1}\left(2 \sqrt{\zeta e^{v} u}\right)\right]
$$

where $u:=\zeta \nu(0) e^{-\kappa_{\nu} T}$. We suggest to use this method when the Feller condition for the variance process is not satisfied. In the second method, we just update the interval $\left[a_{v}^{(j)}, b_{v}^{(j)}\right]$ by subtracting and adding the approximated value for the variance in (6.11) to $a_{v}^{(j)}$ and $b_{v}^{(j)}$, respectively. We suggest to use this method when the Feller condition for the variance process is satisfied.

Once the truncated integration domain $\left[a_{\nu}, b_{\nu}\right]$ has been identified via the above steps, then $V_{2}(0, S(0))$ can be approximated as follows

$$
V_{2}(0, S(0)) \approx V_{3}(0, S(0))=\frac{1}{2^{m_{\nu} / 2}} \sum_{\ell \in \mathcal{L}} c_{\ell} \sum_{n \in \mathbb{N}} d_{n} \int_{a_{\nu}}^{b_{\nu}}\left[\sum_{k_{\nu}=0}^{\left\lceil 2^{m_{\nu}} T\right\rceil} c_{m_{\nu}, k_{\nu}}^{\nu, *}\left(e^{v}\right) g_{\ell, n}\left(\frac{k_{\nu}}{2^{m_{\nu}}}, e^{v}\right)\right] \bar{w}(v) \mathrm{d} v
$$

Then, we consider a partition of the integration interval $\left[a_{\nu}, b_{\nu}\right]$ into $N_{I}$ subintervals, and by the composite trapezoidal rule, we obtain the approximation $V_{4}(0, S(0))$ to $V(0, S(0))$

$$
V_{3}(0, S(0)) \approx V_{4}(0, S(0))=\sum_{\ell \in \mathcal{L}} c_{\ell} \sum_{n \in \mathbb{N}} d_{n} \frac{h}{2} \sum_{l=0}^{N_{I}-1}\left(\mathcal{S}_{m_{\nu}, n}\left(v_{l}\right)+\mathcal{S}_{m_{\nu}}^{\ell, n}\left(v_{l+1}\right)\right)
$$

where

$$
\mathcal{S}_{m_{\nu}}^{\ell, n}(v)=\frac{1}{2^{m_{\nu} / 2}}\left[\sum_{k_{\nu}=0}^{\left\lceil 2^{m_{\nu}} T\right\rceil} c_{m_{\nu}, k_{\nu}}^{\nu, *}\left(e^{v}\right) g_{\ell, n}\left(\frac{k_{\nu}}{2^{m_{\nu}}}, e^{v}\right)\right] \bar{w}(v),
$$

and $h=\frac{b_{v}-a_{v}}{N_{I}}$ and $v_{l}=a_{v}+l h, l=0, \ldots, N_{I}$. Finally, taking $N_{J}$ terms in the infinite series due to jumps, and putting everything together, we have, for the "two-density" treatment,

$$
V_{5}(0, S(0)) \approx V_{4}(0, S(0))=\sum_{\ell \in \mathcal{L}} c_{\ell} \sum_{n=0}^{N_{J}} d_{n} \frac{h}{2} \sum_{l=0}^{N_{I}-1}\left(\mathcal{S}_{m_{\nu}}^{\ell, n}\left(v_{l}\right)+\mathcal{S}_{m_{\nu}}^{\ell, n}\left(v_{l+1}\right)\right)
$$


where $\mathcal{S}_{m_{\nu}}^{\ell, n}(\cdot)$ is defined in (6.14) $, M=m_{d}+m_{f}, a_{s}=0, b_{s}=T, c_{m_{s}, k_{s}}^{s, *}, s=\{d, f\}$, are given in (7.2).

With the "combined-density" treatment, proceeding in a similar fashion, we obtain

$$
V_{5}(0, S(0)) \approx V_{4}(0, S(0))=\frac{e^{-\lambda T}}{2^{m_{c} / 2}} \sum_{k_{c}=\left\lfloor 2^{m_{c}} a_{c}\right\rfloor}^{\left\lceil 2^{m_{c}} b_{c}\right\rceil} c_{m_{c}, k_{c}}^{*} \sum_{n=0}^{N_{J}} \frac{(\lambda T)^{n}}{n !} \frac{h}{2} \sum_{l=0}^{N_{I}-1}\left(\mathcal{S}_{m_{\nu}}^{\ell, n}\left(v_{l}\right)+\mathcal{S}_{m_{\nu}}^{\ell, n}\left(v_{l+1}\right)\right),
$$

where $c_{m_{c}, k_{c}}^{*}$ are defined in (4.16), and $a_{c}=-T$ and $b_{c}=T$.

\section{Error analysis and choice of relevant parameters}

The error arising from the numerical method proposed in this work can be basically divided into two parts. The first part is the approximation carried out for solving the expectations in (3.7) and (3.9) for the "two-density" treatment and the "combined-density" treatment, respectively. The second part concerns the computation of $\mathbb{E}^{\mathbb{Q}}[\cdot]$ described in Section 6. We will focus on the first source of the overall error, since the second has been studied in detail in Dang and Ortiz-Gracia (2018).

The most relevant part in the error analysis when we compute the expectations (3.7) and (3.9) is the recovery of the densities $f_{d}(\cdot), f_{f}(\cdot)$ and $f_{c}(\cdot)$ detailed in Section 4.2 and 4.3 by means of SWIFT method. The error on the recovery of a density from its characteristic function has been extensively studied in Maree et al. (2017) and Dang and Ortiz-Gracia (2018). For sake of completeness, we give a review on this analysis, since it is important for the choice of two relevant parameters of the numerical method.

Let us assume that a certain density function $f$ is well approximated at scale of resolution $m$ in a finite interval $[a, b] \subset \mathbb{R}$. We define $k_{1}:=\left\lfloor 2^{m} a\right\rfloor$ and $k_{2}:=\left\lceil 2^{m} b\right\rceil$. Generally speaking, we aim at approximating $f$ by the following combination of Shannon wavelets

$$
f(x) \approx f_{m}^{*}(x):=\sum_{k=k_{1}}^{k_{2}} c_{m, k}^{*} \varphi_{m, k}(x),
$$

where

$$
c_{m, k}^{*}=\frac{2^{m / 2}}{2^{J-1}} \sum_{j=1}^{2^{J-1}} \Re\left\{\Psi\left(\frac{(2 j-1) \pi 2^{m}}{2^{J}}\right) e^{-\frac{i k \pi(2 j-1)}{2^{J}}}\right\},
$$

and $\Psi(\cdot)$ is the characteristic function associated to $f$. Observe that $[a, b]=[0, T]$ in Section 4.2 and $[a, b]=[-T, T]$ in Section 4.3 . We define the projection error, denoted by $\epsilon_{p}$, as

$$
\epsilon_{p}:=\left|f(x)-\mathcal{P}_{m} f(x)\right|=\left|f(x)-\sum_{k \in \mathbb{Z}} c_{m, k} \varphi_{m, k}(x)\right| .
$$

We also define the truncation error, denoted by $\epsilon_{t}$, as

$$
\epsilon_{t}:=\left|\mathcal{P}_{m} f(x)-f_{m}(x)\right|=\left|\sum_{k \notin\left\{k_{1}, \ldots, k_{2}\right\}} c_{m, k} \varphi_{m, k}(x)\right|
$$

We denote by $\epsilon_{c}$ the error arising from using $c_{m, k}^{*}$ instead of the exact ones $c_{m, k}$. We have,

$$
\epsilon_{c}:=\left|f_{m}(x)-f_{m}^{*}(x)\right|=\left|\sum_{k=k_{1}}^{k_{2}}\left(c_{m, k}-c_{m, k}^{*}\right) \varphi_{m, k}(x)\right| \text {. }
$$


Then, we have,

$$
\left|f(x)-f_{m}^{*}(x)\right| \leq \epsilon_{p}+\epsilon_{t}+\epsilon_{c},
$$

First, we consider $\epsilon_{p}$. The projection $\mathcal{P}_{m} f$ can be written as (Maree et al., 2017)

$$
\mathcal{P}_{m} f(x)=\frac{1}{2 \pi} \int_{-2^{m} \pi}^{2^{m} \pi} \tilde{f}(\xi) e^{i \xi x} \mathrm{~d} \xi=\frac{1}{2 \pi} \int_{-2^{m} \pi}^{2^{m} \pi} \Psi(\xi) e^{-i \xi x} \mathrm{~d} \xi
$$

where

$$
\tilde{f}(\xi):=\int_{\mathbb{R}} f(x) e^{-i \xi x} \mathrm{~d} x .
$$

By definition of the inverse of $\tilde{f}$, we have

$$
f(x)=\frac{1}{2 \pi} \int_{\mathbb{R}} \tilde{f}(\xi) e^{i \xi x} \mathrm{~d} \xi=\frac{1}{2 \pi} \int_{\mathbb{R}} \Psi(\xi) e^{-i \xi x} \mathrm{~d} \xi .
$$

Let

$$
K(v)=\frac{1}{2 \pi} \int_{|\xi|>v}|\Psi(\xi)| \mathrm{d} \xi
$$

then

$$
\epsilon_{p} \leq K\left(2^{m} \pi\right)
$$

Next, we consider the truncation error $\epsilon_{t}$. We observe that

$$
\epsilon_{t}=\left|\mathcal{P}_{m} f(x)-f_{m}(x)\right| \leq 2^{m / 2} \sum_{k \notin\left\{k_{1}, \ldots, k_{2}\right\}}\left|c_{m, k}\right|
$$

since $\left|\varphi_{m, k}(x)\right| \leq 2^{m / 2}$. If we take into account the definition of $c_{m, k}$ in (4.2) and the fact that within the present work $f$ is compactly supported in $[a, b]$, then the truncation error can be neglected.

Finally, we consider $\epsilon_{c}$. The numerical error can be estimated as

$$
\epsilon_{c} \leq \sum_{k=k_{1}}^{k_{2}}\left|c_{m, k}-c_{m, k}^{*}\right|\left|\varphi_{m, k}(x)\right| \leq 2^{m / 2} \sum_{k=k_{1}}^{k_{2}}\left|c_{m, k}-c_{m, k}^{*}\right| .
$$

The coefficients approximation error is studied in Theorem 1 of Ortiz-Gracia and Oosterlee (2016) and we recall here as follows.

Theorem 7.1 (Theorem 1 of Ortiz-Gracia and Oosterlee (2016)). Let $F(x)$ be the distribution function of a random variable $X$ and define $H(x):=F(-x)+1-F(x)$. Let $\mathcal{A}>0$ be a constant such that $H(\mathcal{A})<\epsilon$, for $\epsilon>0$. Define $M_{m, k}:=\max \left(\left|2^{m} \mathcal{A}-k\right|,\left|2^{m} \mathcal{A}+k\right|\right)$ and consider $J \geq \log _{2}\left(\pi M_{m, k}\right)$. Then

$$
\left|c_{m, k}-c_{m, k}^{*}\right| \leq 2^{m / 2}\left(2 \epsilon+\sqrt{2 \mathcal{A}}\|f\|_{2} \frac{\left(\pi M_{m, k}\right)^{2}}{2^{2(J+1)}-\left(\pi M_{m, k}\right)^{2}}\right),
$$

and $\lim _{J \rightarrow+\infty} c_{m, k}^{*}=c_{m, k}$.

Within the present work, $F$ represents the distribution function of the compactly supported density $f$ and then, if we define $\mathcal{A}:=\max (|a|,|b|)$, we have $H(\mathcal{A})=0$. We can apply Theorem 7.1 with $J \geq \log _{2}\left(\pi M_{m}\right)$, where $M_{m}:=\max _{k_{1}<k<k_{2}} M_{m, k}$. Finally

$$
\epsilon_{c} \leq 2^{m / 2} \sum_{k=k_{1}}^{k_{2}}\left|c_{m, k}-c_{m, k}^{*}\right| \leq 2^{m}\left(k_{2}-k_{1}+1\right) \sqrt{2 \mathcal{A}}\|f\|_{2} \frac{\left(\pi M_{m}\right)^{2}}{2^{2(\jmath+1)}-\left(\pi M_{m}\right)^{2}} .
$$

From (7.2), we note that the two parameters, namely the level of resolution $m$ and the truncation parameter $J$, need to be determined before this inversion. In this section, we discuss how to select $m$ and $J$. From the above paragraph we know that we can pick $J \geq \log _{2}\left(\pi M_{m}\right)$ once an appropriate 

simplicity, we only show the "combined-density" treatment.

value for $m$ has been selected, so we first discuss how to select an appropriate value for $m$. We proceed by finding $m$ such that the projection error $\epsilon_{p}$, defined in (7.3), is below a pre-determined tolerance tol. We denote by $\epsilon_{p}^{(m)}$ an approximation to $\epsilon_{p}$, given the level of resolution $m$. From the bound (7.9), together with (7.8), we approximate $\epsilon_{p}^{(m)}$ by the rough but easy to compute expression

$$
\epsilon_{p}^{(m)}:=\frac{1}{2 \pi}\left(\left|\Psi\left(-2^{m} \pi\right)\right|+\left|\Psi\left(2^{m} \pi\right)\right|\right) .
$$

We can find the level of resolution $m$ by iteratively computing the first $m$ such that $\epsilon_{p}^{(m)} \leq$ tol. When the parameter $m$ has been selected by the above-described procedure, we consider $J=\log _{2}\left(\pi M_{m}\right)$.

Finally, it is worth remarking that once the relevant parameters $m$ and $J$ have been selected, we can compute very fast the coefficients in (7.2) by following an FFT algorithm. An algorithm to approximate $V(S(0), 0, \cdot)$ using the proposed Shannon wavelet method is given in Algorithm [7.1. For

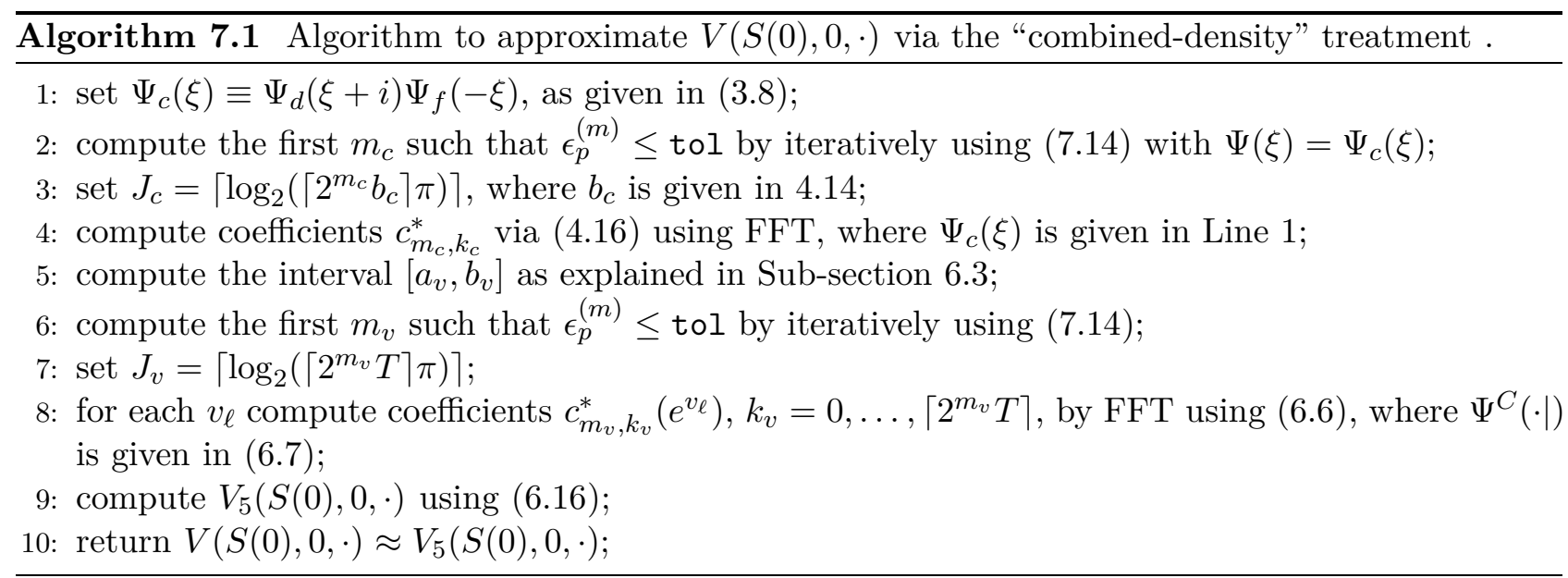

\section{Numerical experiments}

In this section, we present selected numerical results to illustrate the performance of the proposed method. We consider both the pure-diffusion and jump-extended versions of the four-factor model in which both the domestic and foreign interest rates follow the one-factor CIR dynamics. These two versions are hereafter referred to as Heston-1CIR and jump-extended Heston-1CIR. We also consider a six-factor model in which both the interest rates follow two-factor CIR dynamics, the pure-diffusion and jump-extended versions of which hereafter are respectively referred to as Heston-2CIR and jumpextended Heston-2CIR.

In determining the truncated integration interval $\left[a_{v}, b_{v}\right]$ for the log-variance density, we consider $\epsilon_{\mathrm{tol}}=10^{-6}$, and follow the procedure explained in Section 6, where a Newton search is used when the Feller condition is not satisfied, and the alternative method otherwise.

To obtain benchmark solutions in the case of no jumps, we use the antithetic multi-level MC method, developed in Giles and Szpruch (2014). We hereafter refer to this method as anti-mlMC. To simulate the CIR processes, namely the interest rates and the variance, we use the Lamperti-BackwardEuler timestepping method that preserves the positivity of the original dynamics (2.4), and has a good strong convergence property, recently established in Neuenkirch and Szpruch (2014). The anti-mlMC method can achieve the overall complexity $\mathcal{O}\left(\epsilon^{-2}\right)$ for a root-mean-square error (RMSE) of $\epsilon$ without simulating iterated Itô integrals, also known as Lévy areas, which is usually very slow. To handle the jumps, we extend the anti-mlMC method by noting that, since the option is not path-dependent, the overall jump effects on the spot FX rate can be evaluated separately at time $T$, and be taken into account at that time. 
All results in this paper were obtained using MATLAB 2017. Comparable optimized code in $\mathrm{C} / \mathrm{C}++$ would likely run significantly faster. Nonetheless, the presented timing results presented below already indicate the significant efficiency of the proposed Shannon wavelet method.

\subsection{Estimating technique for the supports of $f_{s}(\cdot), s=\{d, f\}$}

As discussed in Cozma and Reisinger (2017), calibrated parameters of CIR interest rate processes typically satisfy the Feller condition, namely $2 \kappa_{(\cdot)} \theta_{(\cdot)}>\sigma_{(\cdot)}^{2}$. However, this condition may not be satisfied for the variance process. For illustrating purposes, we include Table 8.1 (Table 2 from Cozma and Reisinger (2017)) that contains calibrated interest rate CIR parameters from different sources of real market data. Specifically, the sources of data are: 3-month US Treasury bill yield between January 1964 - December 1998 (Driffill et al., 2003), US Treasury bill yield between Octo-

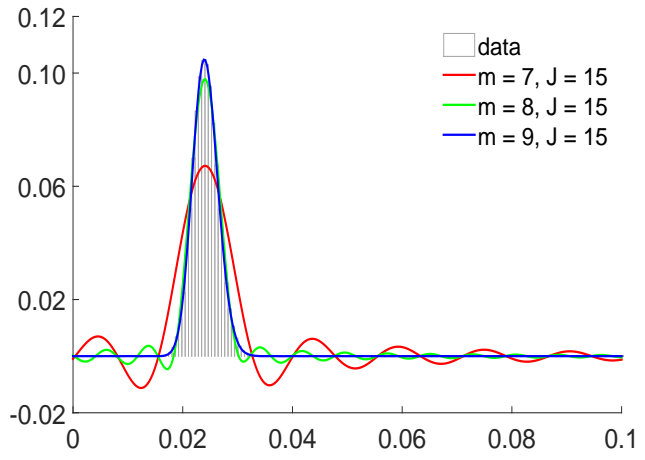

Figure 8.1: Recovered densities of the time-integrated CIR processes for different levels of resolution $m$. ber 1982 - April 2011 (Erismann, 2011), to the Euro ATM caps volatility curve on 17 January 2000 (Brigo and Mercurio, 2006), Euro OverNight Index Average between 1 January 2008 - 6 October 2008 (Lafférs, 2009), and historical data for Euro between 1 January 2001 - 1 September 2011 (Amin, 2012).

\begin{tabular}{|l|c|c|c|}
\hline & $\kappa_{(\cdot)}$ & $\theta_{(\cdot)}$ & $\sigma_{(\cdot)}$ \\
\hline Driffill et al. (2003) & 0.0684 & 0.0161 & 0.0177 \\
Erismann 2011) & 0.1104 & 0.0509 & 0.0498 \\
Brigo and Mercurio (2006) & 0.3945 & 0.2713 & 0.0545 \\
Lafférs (2009) & 0.2820 & 0.0411 & 0.0058 \\
Amin (2012) & 0.1990 & 0.0497 & 0.0354 \\
\hline
\end{tabular}

Table 8.1: Typical calibrated domestic and foreign interest rate CIR parameters from different sources. The Feller condition $2 \kappa_{(\cdot)} \theta_{(\cdot)}>\sigma_{(\cdot)}^{2}$ is satisfied.

Motivated by these observations, we will now investigate the densities of the time-integrated onefactor CIR processes recovered by SWIFT method. We take $\kappa_{(\cdot)}=0.0684, \theta_{(\cdot)}=0.0161$, and $\sigma_{(\cdot)}=$ 0.0177 from the Table 8.1. We also show a histogram of the Monte-Carlo generated time-integrated interest rates for these parameters. In this Monte-Carlo simulation, $10^{4}$ timesteps and $10^{6}$ samples are used. We observe from Figure 8.1 that the right tail of the density of the time-integrated interest rate processes appears to decay to zero rapidly. As such, given a right level of resolution $m$, instead of using $\left[a_{s}, b_{s}\right]=[0, T]$ for the support of the $f_{s}(\cdot), s=\{d, f\}$, a carefully estimated smaller support of the form $\left[0, b_{s}\right], b_{s}<T$, that has negligible loss of density mass could be employed so that the efficiency of the Shannon wavelet method could be increased (i.e. significantly reduce the computational time without affecting the accuracy of the numerical solutions). Once $b_{s}, s=\{d, f\}$, has been found, an estimated support for $f_{c}(\cdot)$ can then be computed using formula (4.14).

Motivated by this, we will investigate the following problem: given the level of resolution $m$, estimate the support of $f_{s}(\cdot), s=\{d, f\}$, so that the loss of density mass is less than some small tolerance. Specifically, given $m_{s}$, we find $b_{s} \in(0, T]$, such that

$$
1-\int_{0}^{b_{s}} \hat{f}_{s, m_{s}}^{*}(t) \mathrm{d} t=\text { tolerance }
$$


where $\hat{f}_{s, m_{s}}^{*}$ is given by

$$
\hat{f}_{s, m_{s}}^{*}(t)=\sum_{k_{s}=0}^{\left\lceil 2^{m_{s}} \times b_{s}\right\rceil} \hat{c}_{m_{s}, k_{s}}^{s, *} \varphi_{m_{s}, k_{s}}(t)
$$

Here,

$$
\hat{c}_{m_{s}, k_{s}}^{s, *}=\frac{2^{m_{s} / 2}}{2^{\hat{J}_{s}-1}} \sum_{j_{s}=1}^{2^{\hat{J}_{s}-1}} \Re\left\{\Psi_{s}\left(\frac{\left(2 j_{s}-1\right) \pi 2^{m_{s}}}{2^{\hat{J}_{s}}}\right) e^{-\frac{i k_{s} \pi\left(2 j_{s}-1\right)}{2^{\hat{J}_{s}}}}\right\},
$$

with $\hat{J}_{s}=\left\lceil\log _{2}\left(\left\lceil 2^{m} b_{s}\right\rceil \pi\right)\right\rceil$, and $\Psi_{s}$ is the known characteristic function in (3.6). We note that Equations (8.1) and (8.2) come from (4.6) and (7.2), respectively. This problem can be solved using a root finding technique, such as a Newton method or the bisection method. In our experiments, very quick convergence can be achieved in a small number of iterations with the bisection method.

\subsection{Heston-1CIR models}

For experiments in this subsection, the parameters are presented in Table 8.2. We note that the parameters of the interest rates and the variance are taken from Cozma and Reisinger (2017). For the

\begin{tabular}{|c|c|c|c|c|c|c|c|c|c|}
\hline$r_{d}$ & $\begin{array}{c}X_{1}(0) \\
0.0524\end{array}$ & $\begin{array}{c}\kappa_{d_{1}} \\
1.8341\end{array}$ & $\begin{array}{c}\theta_{d_{1}} \\
0.0475\end{array}$ & $\begin{array}{c}\sigma_{d_{1}} \\
0.0352\end{array}$ & $r_{f}$ & $\begin{array}{c}Y_{1}(0) \\
0.0291\end{array}$ & $\begin{array}{c}\kappa_{f_{1}} \\
0.32\end{array}$ & $\begin{array}{c}\theta_{f_{1}} \\
0.0248\end{array}$ & $\begin{array}{c}\sigma_{f_{1}} \\
0.0317\end{array}$ \\
\hline$\nu$ & $\begin{array}{c}\nu(0) \\
0.0275\end{array}$ & $\begin{array}{c}\kappa_{\nu} \\
1.70\end{array}$ & $\begin{array}{c}\bar{\nu} \\
0.0232\end{array}$ & $\begin{array}{c}\sigma_{\nu} \\
0.1500\end{array}$ & jump & $\begin{array}{c}\lambda \\
0.2\end{array}$ & $\begin{array}{c}\tilde{\mu} \\
-0.08\end{array}$ & $\begin{array}{c}\tilde{\sigma} \\
0.3\end{array}$ & \\
\hline others & $\begin{array}{c}S(0) \\
100\end{array}$ & $\begin{array}{c}K \\
100\end{array}$ & $\begin{array}{l}\rho_{S, \nu} \\
-0.1\end{array}$ & & & & & & \\
\hline
\end{tabular}
jump-extended case, the parameters for the normal jump amplitude are taken from Dang (2017).

Table 8.2: Parameters for experiments with the Heston-1CIR models.

\subsubsection{Heston-1CIR model}

In this test, we consider a European call option under the Heston-CIR dynamics for different maturities, namely $T=\{0.25,1,3\}$. We will also compare the efficiency between the "two-density" and and the "combined-density" treatments, as discussed in Subsection 4.3. But first, we study the effects of the levels of resolution $m_{d}$ (time-integrated domestic rate density), $m_{f}$ (time-integrated foreign rate density), $m_{c}$ (combined-density), and $m_{\nu}$ (conditional time-integrated variance density), as well as the number of subintervals $N_{I}$ for the composite trapezoidal rule on the computed prices of the option.

For simplicity, we choose $m_{d}=m_{f}=m_{c}=m_{\nu}=m$. For each value of $m$, we also report the corresponding projection error, generically denoted by $\epsilon_{p}^{(m)}$, defined in Section 7 (note that $\epsilon_{p}^{(m)}$ is independent of $N_{I}$ ). In the case of interest rates (domestic, foreign, and combined), we approximate the projection error by the following formula:

$$
\epsilon_{p}^{(m)}:=\frac{1}{2 \pi}\left(\left|\Psi\left(-2^{m} \pi\right)\right|+\left|\Psi\left(2^{m} \pi\right)\right|\right) .
$$

For the variance factor, we use

$$
\epsilon_{p}^{(m)}:=\frac{1}{2 \pi} \max _{v}\left(\left|\Psi\left(-2^{m} \pi \mid e^{v}\right)\right|+\left|\Psi\left(2^{m} \pi \mid e^{v}\right)\right|\right),
$$

where $v=\ln (\nu(T))$.

Table 8.3 presents selected numerical results when the "combined-density" treatment is used, i.e. $\Psi_{c}(\cdot)=\Psi_{d}(\cdot) \Psi_{f}(\cdot)$. We note that the benchmark option prices are obtained by the anti-mlMC with 


\begin{tabular}{|c|c|c|c|c|c|c|c|c|c|c|c|c|c|}
\hline \multirow{3}{*}{$\overrightarrow{N_{I}}$} & & \multicolumn{4}{|c|}{$T=0.25$} & \multicolumn{4}{|c|}{$T=1$} & \multicolumn{4}{|c|}{$T=3$} \\
\hline & $m$ & \multicolumn{2}{|c|}{$\epsilon_{p}^{(m)}$} & \multirow{2}{*}{$\begin{array}{l}\text { abs. } \\
\text { error }\end{array}$} & \multirow{2}{*}{$\begin{array}{l}\text { time } \\
\text { (sec.) }\end{array}$} & \multicolumn{2}{|c|}{$\epsilon_{p}^{(m)}$} & \multirow{2}{*}{$\begin{array}{l}\text { abs. } \\
\text { error }\end{array}$} & \multirow{2}{*}{$\begin{array}{c}\text { time } \\
\text { (sec.) }\end{array}$} & \multicolumn{2}{|c|}{$\epsilon_{p}^{(m)}$} & \multirow{2}{*}{$\begin{array}{l}\text { abs. } \\
\text { error }\end{array}$} & \multirow{2}{*}{$\begin{array}{c}\text { time } \\
\text { (sec.) }\end{array}$} \\
\hline & & $r$ & $\nu$ & & & $r$ & $\nu$ & & & $r$ & $\nu$ & & \\
\hline \multirow{4}{*}{15} & 7 & $3.0 \mathrm{e}-01$ & $3.1 \mathrm{e}-01$ & $9.2 \mathrm{e}-02$ & 0.07 & $3.6 \mathrm{e}-02$ & $1.0 \mathrm{e}-01$ & $1.5 \mathrm{e}-02$ & 0.14 & $4.1 \mathrm{e}-14$ & $3.2 \mathrm{e}-04$ & $1.5 \mathrm{e}-04$ & 0.53 \\
\hline & 8 & $2.7 \mathrm{e}-01$ & $2.9 \mathrm{e}-01$ & $8.9 \mathrm{e}-02$ & 0.09 & $8.0 \mathrm{e}-05$ & $1.1 \mathrm{e}-02$ & $5.0 \mathrm{e}-04$ & 0.28 & $7.5 \mathrm{e}-31$ & $5.7 \mathrm{e}-07$ & $1.5 \mathrm{e}-04$ & 1.92 \\
\hline & 9 & $1.7 \mathrm{e}-01$ & $2.3 \mathrm{e}-01$ & $4.7 \mathrm{e}-03$ & 0.12 & $1.9 \mathrm{e}-14$ & $2.1 \mathrm{e}-04$ & $4.8 \mathrm{e}-04$ & 0.85 & $2.1 \mathrm{e}-51$ & $2.9 \mathrm{e}-11$ & $1.6 \mathrm{e}-04$ & 7.79 \\
\hline & 10 & $2.8 \mathrm{e}-02$ & $7.8 \mathrm{e}-02$ & $6.8 \mathrm{e}-04$ & 0.25 & $1.9 \mathrm{e}-14$ & $4.7 \mathrm{e}-07$ & $4.8 \mathrm{e}-04$ & 3.03 & $5.0 \mathrm{e}-78$ & $1.2 \mathrm{e}-17$ & $1.6 \mathrm{e}-04$ & 27.54 \\
\hline \multirow{4}{*}{25} & 7 & & & $9.2 \mathrm{e}-02$ & 0.10 & & & $1.5 \mathrm{e}-02$ & 0.16 & & & $1.5 \mathrm{e}-04$ & 0.85 \\
\hline & 8 & & & $8.9 \mathrm{e}-02$ & 0.14 & & & $4.4 \mathrm{e}-04$ & 0.40 & & & $1.5 \mathrm{e}-04$ & 3.21 \\
\hline & 9 & & & $4.9 \mathrm{e}-03$ & 0.16 & & & $4.8 \mathrm{e}-04$ & 1.43 & & & $1.6 \mathrm{e}-04$ & 11.38 \\
\hline & 10 & & & $6.7 \mathrm{e}-04$ & 0.45 & & & $4.9 \mathrm{e}-04$ & 4.84 & & & $1.6 \mathrm{e}-04$ & 42.96 \\
\hline
\end{tabular}

Table 8.3: European call option under the Heston-1CIR model with different maturities using parameters in Table 8.2. The "combined-density" treatment is used. For this test, $m_{c}=m_{\nu}=m$, and the supports respectively are $[-T, T]$ and $[0, T]$ for $f_{c}(\cdot)$ and $f(\cdot \mid y)$. The benchmark solutions obtained by the anti-mlMC method $\left(\mathrm{RMSE}=10^{-3}\right.$ ) are: 3.50363381 (std. dev. $\approx 7.1 \mathrm{e}-04$ ) for $T=0.25 ; 7.21360895$ (std. dev. $\approx 7.1 \mathrm{e}-04$ ) for $T=1$; and 12.93507573 (std. dev. $\approx 7.1 \mathrm{e}-04$ ) for $T=3$.

the RSME set to $10^{-3}$, and hence, the standard deviations in the benchmark option prices all are $\leq \frac{10^{-3}}{\sqrt{2}} \approx 7.1 \times 10^{-04}$, as expected from analysis of multi-level MC methods (Giles, 2008). We make the following observations.

- Across different values of $N_{I}$, for a given $m$, an increase in $N_{I}$ does not appear to improve the accuracy. This seems to hold true for all maturities. For example, for $m=7$ and $T=0.25$ the absolute errors are 9.2e- 02 across all levels of $N_{I}$; for $m=8$ and $T=3$, the absolute errors are approximately $1.6 \mathrm{e}-4$ for all levels of $N_{I}$.

- With the above observation in mind, we now focus on the effects of $m$ on the accuracy when $N_{I}=15$. We observe that, for the short maturity case, namely $T=0.25$, the absolute error decreases when the level of resolution $m$ increases (e.g. from 9.2e-2 when $m=7$ down to $6.8 \mathrm{e}-4$ when $m=10$, at which the projection errors are 2.8e-02 and 7.8e-02 for the "combined-density" $r$ and the variance $\nu$, respectively.

For longer maturities $T=\{1,3\}$, the absolute errors stay approximately the same when $m$ is sufficiently large. In particular, for $T=1$, the error is $1.5 \mathrm{e}-02$ when $m=7$, but decreases rapidly to around 5.0e-04 for $m=8,9,10$. For $T=3$, the absolute error stays around 1.6e-04 for all levels of resolution $m$ considered. Moreover, compared to the benchmark solutions, the price computed by the Shannon wavelet method is already accurate with $m=8$ for the case $T=1$ (with the error being 5.0e-04), and with $m=7$ for the case $T=3$ (with the error being 1.6e-04). We also note that the corresponding projection errors for these two longer maturities are much smaller compared to the case $T=0.25$.

Based on these results, with the "combined-density" treatment, we will use $N_{I}=15$ and the tol $=$ $10^{-02}$ in estimating the level resolution $m$, i.e. find the first level of resolution $m$ such that for $\epsilon_{p}^{(m)} \leq$ tol, as discussed in Section 7 . We emphasize that with this choice of $m$ and $N_{I}=15$, the prices under the Heston-1CIR model are obtained very quickly. Specifically, it took 0.25 seconds for $T=0.25(m=10), 0.28$ seconds for $T=1(m=8)$, and 0.53 seconds for $T=3(m=7)$. For the reader's convenience, these results are grayed out in Table 8.3 .

\section{Efficiency comparison: "two-density" vs. "combined-density" treatments}

Next, we compare the efficiency between the "two-density" and the "combined-density" treatments. In Table 8.4, we present selected numerical results of these two treatments, with absolute errors and 

timing results for the "combined-density" treatment being copied from Table 8.3 for the reader's convenience. Note that we do not report the projection errors for the time-integrated variance process under the "two-density" treatment, as they are the same with those when the "combined-density" treatment is used (see Table 8.3). We observe from Table 8.4 that the "combined-density" treatment

\begin{tabular}{|c|c|c|c|c|c|c|c|c|c|c|c|c|c|}
\hline & \multicolumn{6}{|c|}{$T=1$} & \multicolumn{6}{|c|}{$T=3$} \\
\hline & & \multicolumn{4}{|c|}{$\begin{array}{c}\text { two } \\
\text { density }\end{array}$} & \multicolumn{2}{|c|}{$\begin{array}{c}\text { combined } \\
\text { density }\end{array}$} & \multicolumn{4}{|c|}{$\begin{array}{c}\text { two } \\
\text { density }\end{array}$} & \multicolumn{2}{|c|}{$\begin{array}{c}\text { combined } \\
\text { density }\end{array}$} \\
\hline \multirow{2}{*}{$N_{I}$} & \multirow[t]{2}{*}{$m$} & \multicolumn{2}{|c|}{$\epsilon_{p}^{(m)}$} & \multirow{2}{*}{$\begin{array}{l}\text { abs. } \\
\text { error }\end{array}$} & \multirow{2}{*}{$\begin{array}{r}\text { time } \\
\text { (sec.) }\end{array}$} & \multirow{2}{*}{$\begin{array}{l}\text { abs. } \\
\text { error }\end{array}$} & \multirow{2}{*}{$\begin{array}{l}\text { time } \\
\text { (sec.) }\end{array}$} & \multicolumn{2}{|c|}{$\epsilon_{p}^{(m)}$} & \multirow{2}{*}{$\begin{array}{l}\text { abs. } \\
\text { error }\end{array}$} & \multirow{2}{*}{$\begin{array}{l}\text { time } \\
\text { (sec.) }\end{array}$} & \multirow{2}{*}{$\begin{array}{l}\text { abs. } \\
\text { error }\end{array}$} & \multirow{2}{*}{\begin{tabular}{|l} 
time \\
(sec.)
\end{tabular}} \\
\hline & & $r_{d}$ & $r_{f}$ & & & & & $r_{d}$ & $r_{f}$ & & & & \\
\hline & 7 & $7.1 \mathrm{e}-02$ & $1.7 \mathrm{e}-01$ & $1.3 \mathrm{e}-01$ & 3.06 & $1.5 \mathrm{e}-02$ & 0.14 & $2.6 \mathrm{e}-10$ & $5.2 \mathrm{e}-05$ & $2.1 \mathrm{e}-04$ & 93.72 & $1.5 \mathrm{e}-04$ & \begin{tabular}{|l|l}
0.53 \\
\end{tabular} \\
\hline 15 & 8 & $9.4 \mathrm{e}-04$ & $2.8 \mathrm{e}-02$ & $6.3 \mathrm{e}-03$ & 21.32 & $5.0 \mathrm{e}-04$ & 0.28 & $8.7 \mathrm{e}-21$ & $2.7 \mathrm{e}-11$ & $1.8 \mathrm{e}-04$ & $>1000$ & $1.5 \mathrm{e}-04$ & 1.92 \\
\hline
\end{tabular}

Table 8.4: Efficiency comparison between the "two-density" and the "combined-density" treatments. European call option under the Heston-1CIR model using parameters in Table 8.2. The results of the "combined-density" treatment are copied from Table 8.3. For the two-density treatment, $m_{d}=m_{f}=$ $m_{\nu}=m$, and the support $[0, T]$ is used for $f_{d}(\cdot), f_{f}(\cdot)$, and $f(\cdot \mid y)$. The benchmark solutions obtained by the anti-mlMC $\left(\mathrm{RMSE}=10^{-3}\right)$, are: 7.21360895 (std. dev. $\approx 7.1 \mathrm{e}-04$ ) for $T=1$; and 12.93507573 (std. dev. $\approx 7.1 \mathrm{e}-04$ ) for $T=3$.

is significantly more efficient than the "two-density" one. For example, when $T=1$, the combineddensity treatment can achieve an absolute error of 5.0e-04 in only 0.28 seconds, while, even with 21.32 seconds, the "two-density" treatment can only achieve an absolute error of $6.3 \mathrm{e}-03$. This means the "combined-density" treatment offers approximately two to three orders of magnitude improvement in computational efficiency over the "two-density" in this case. When $T=3$, the improvement in computational efficiency offered by the "combined-density" treatment is also between two and three orders. Such superiority of the "combined-density" treatment over the "two-density" treatment is expected, as previously noted in Remark 4.1. As such, for the rest of the experiments in the paper, we will only present numerical results of the "combined-density" treatment, but we emphasize that a significantly better efficiency of the "combined-density" treatment is observed in all test cases.

\section{Estimation of support of $f_{c}(\cdot)$}

Finally, we investigate the effects on the computational efficiency of the estimating technique discussed in Subsection 8.1 of the support of $f_{c}(\cdot)$ ("combined-density" treatment).

In Table 8.5 (a), we show selected numerical results of the same European call options for the experiment reported in Table 8.3 , but this time, instead of using the full support $[-T, T]$ for $f_{c}(\cdot)$, we use the support estimated by the technique described in Subsection 8.1, with the tolerance being $10^{-02}$. We observe that with this technique, we can achieve virtually the same prices with approximately onefourth of the computational times $(0.13 / 0.53 \approx 1 / 4$ while the absolute change is about $1.0 \mathrm{e}-06)$.

To further investigate possible computational savings that this technique could offer, we experiment with relatively longer maturities. In Table 8.5 (b), we report selected numerical results when pricing a European put option with maturities $T=\{5,8,10\}$. We first note that the prices produced by the Shannon wavelet method with the estimated support or full support (e.g. $[-T, T]$ ) are (i) virtually the same, and (ii) in excellent agreement with the benchmark prices obtained by the anti-mlMC method (with RMSE $=3 \times 10^{-3}$ ). (The standard deviations in the benchmark option prices all are $\leq \frac{3 \times 10^{-3}}{\sqrt{2}} \approx 0.0021$, as expected.) Moreover, we observe that the support estimating technique offers significant computational savings, cutting down the computational times by a factor of approximately seven (for example, $1.57 / 0.2 \approx 7$, and $5.11 / 0.7 \approx 7$ ). With this estimating technique, the efficiency of the Shannon wavelet method is substantial. Compared to benchmark prices, it is able to price a 
10-year option with a relative error of about $0.02 \%$ (e.g. $(6.8345-6.8330) / 6.8330$ ) in about only 0.7 seconds (see grayed out results in Table 8.5 b).

\begin{tabular}{|c|c|c|c|c|c|c|c|c|c|}
\hline \multirow[b]{2}{*}{$T$} & \multirow[b]{2}{*}{$m$} & \multicolumn{5}{|c|}{ estimated support } & \multicolumn{2}{|c|}{ full support } & \multirow{2}{*}{$\begin{array}{c}\text { abs. } \\
\text { change } \\
\mid(\mathrm{a}) \text { - (b) }\end{array}$} \\
\hline & & $b_{d}$ & $b_{f}$ & {$\left[a_{c}, b_{c}\right]$} & $\begin{array}{r}\text { price } \\
\text { (a) }\end{array}$ & $\begin{array}{l}\text { time } \\
\text { (sec.) }\end{array}$ & $\begin{array}{r}\text { price } \\
\text { (b) }\end{array}$ & $\begin{array}{l}\text { time } \\
\text { (sec.) }\end{array}$ & \\
\hline & & & & & & & \multicolumn{2}{|c|}{ from Table 8.3} & \\
\hline 0.25 & 10 & 0.015 & 0.008 & {$[-0.016,0.016]$} & 3.5034 & 0.07 & 3.5029 & 0.25 & $\approx 5.0 \mathrm{e}-04$ \\
\hline 1 & 8 & 0.062 & 0.036 & {$[-0.062,0.062]$} & 7.2131 & 0.08 & 7.2131 & 0.28 & $\approx 2.4 \mathrm{e}-05$ \\
\hline 3 & 7 & 0.204 & 0.114 & {$[-0.204,0.204]$} & 12.9349 & 0.13 & 12.9349 & 0.53 & $\approx 1.0 \mathrm{e}-06$ \\
\hline
\end{tabular}

(a) Call options with parameters in Table 8.2. "combined-density" treatment, $N_{I}=15$, and dynamic estimation for supports with tolerance $10^{-02}$. The benchmark solutions for the European call option, obtained by the anti-mlMC method (with RMSE $=10^{-3}$ ) are: 3.50363381 (std. dev. $\approx 7.1 \mathrm{e}-04$ ) for $T=0.25 ; 7.21360895$ (std. dev. $\approx 7.1 \mathrm{e}-04$ ) for $T=1$; and 12.93507573 (std. dev. $\approx 7.1 \mathrm{e}-04$ ) for $T=3$.

\begin{tabular}{|c|c|c|c|c|c|c|c|c|c|}
\hline$T$ & & \multicolumn{4}{|c|}{ estimated support } & \multicolumn{2}{c|}{ full support } & abs. \\
& $m$ & $b_{d}$ & $b_{f}$ & {$\left[a_{c}, b_{c}\right]$} & $\begin{array}{c}\text { price } \\
(\mathrm{a})\end{array}$ & $\begin{array}{c}\text { time } \\
(\mathrm{sec} .)\end{array}$ & $\begin{array}{c}\text { price } \\
(\mathrm{b})\end{array}$ & $\begin{array}{c}\text { time } \\
(\mathrm{sec} .)\end{array}$ & $\begin{array}{c}\text { change } \\
|(\mathrm{a})-(\mathrm{b})|\end{array}$ \\
\hline 5 & 7 & 0.3501 & 0.1907 & {$[-0.3501,0.3501]$} & 7.1052 & 0.20 & 7.1052 & 1.57 & $<1.0 \mathrm{e}-06$ \\
\hline 8 & 7 & 0.5603 & 0.2956 & {$[-0.5603,0.5603]$} & 7.0697 & 0.53 & 7.0697 & 3.40 & $<1.0 \mathrm{e}-06$ \\
\hline 10 & 7 & 0.7004 & 0.3580 & {$[-0.7004,0.7004]$} & 6.8345 & 0.70 & 6.8345 & 5.11 & $<1.0 \mathrm{e}-06$ \\
\hline
\end{tabular}

(b) Put options with parameters in Table 8.2 "combined-density" treatment, $N_{I}=15$, and dynamic estimation for supports with tolerance $10^{-02}$. The benchmark solutions obtained by the anti-mlMC method (with RMSE $=3 \times 10^{-3}$ ) are: 7.1061 (std. dev. $\left.\approx 2.1 \mathrm{e}-03,95 \%[7.1021,7.1103]\right)$ for $T=5 ; 7.0678$ (std. dev. $\approx 2.1 \mathrm{e}-03,95 \%$ $[7.0648,7.0730]$ ) for $T=8$; and 6.8330 (std. dev. $\approx 2.1 \mathrm{e}-03,95 \%$ CI $[6.8289,6.8371]$ ) for $T=10$.

Table 8.5: Effects on computational efficiency of the technique estimating the support of $f_{c}(\cdot)$ via the tolerance $10^{-02}$.

We conclude this subsection by noting that, due to the significant computational savings of the "combined-density" treatment and the estimating technique for the support of $f_{c}(\cdot)$, we will adopt to implement them in all the remaining experiments.

\subsubsection{Jump-extended Heston-1CIR model}

In Table 8.6, we present selected numerical results of pricing a European call and put options under the jump-extended Heston-1CIR model, respectively. In this experiment, we use $N_{J}=8$ in (6.16), i.e. the first 9 terms of the series due to jumps, for which the truncation error of the series is already less than $10^{-6}$. Again, we note excellent agreement between the benchmark solutions obtained by the anti-mlMC method and those produced by the Shannon wavelet method. In addition, the performance of the method is also impressive.

\subsection{Heston-2CIR models}

Finally, we consider the valuation of a European option under the Heston-2CIR models. For experiments in this subsection, we use the parameters presented in Table 8.7. We note that the calibrated parameters of the two-factor CIR interest rate processes from (Chen and Scott, 1992, 2003). In addition, we consider two different set of parameters for the variance

- Set 1: $\nu(0)=0.0275, \kappa_{\nu}=1.7, \bar{\nu}=0.0232, \sigma_{\nu}=0.15$, which are similar to those in Table 8.2. For this set of parameters, the Feller's condition is satisfied

- Set 2: $\nu(0)=0.2, \kappa_{\nu}=0.1, \bar{\nu}=0.6, \sigma_{\nu}=0.5$ from Dang and Ortiz-Gracia (2018), for which Feller's condition is not satisfied. 


\begin{tabular}{|c|c|c|c|r|c|c|c|}
\cline { 3 - 8 } \multicolumn{2}{|c|}{} & \multicolumn{2}{|c|}{ anti-mlMC } & \multicolumn{4}{|c|}{ Shannon wavelets } \\
\hline \begin{tabular}{c} 
(years) \\
\hline
\end{tabular} & $m$ & (price, std. dev. ) & $95 \%$ CI & price & $\begin{array}{c}\text { abs. } \\
\text { error }\end{array}$ & $\begin{array}{c}\text { rel. } \\
\text { error }(\%)\end{array}$ & $\begin{array}{c}\text { time } \\
(\text { sec.) }\end{array}$ \\
\hline 0.25 & 10 & $(3.9507,7.1 \mathrm{e}-4)$ & {$[3.9493,3.9520]$} & 3.9497 & $7.0 \mathrm{e}-04$ & $\approx 0.01$ & 0.24 \\
1 & 8 & $(8.5535,7.1 \mathrm{e}-4)$ & {$[8.5521,8.5549]$} & 8.5543 & $9.0 \mathrm{e}-04$ & $\approx 0.01$ & 0.26 \\
3 & 7 & $(15.5424,7.1 \mathrm{e}-4)$ & {$[15.5394,15.5421]$} & 15.5416 & $8.0 \mathrm{e}-04$ & $\approx 0.01$ & 0.60 \\
\hline
\end{tabular}

(a) Call options prices, the benchmark prices obtained by the anti-mlMC method $\left(\mathrm{RMSE}=3 \times 10^{-03}\right.$ )

\begin{tabular}{|c|c|c|c|c|c|c|c|}
\cline { 3 - 8 } \multicolumn{2}{c|}{} & \multicolumn{3}{c|}{ anti-mlMC } & \multicolumn{4}{c|}{ Shannon wavelets } \\
\hline $\begin{array}{c}T \\
\text { (years) }\end{array}$ & $m$ & (price, std. dev. ) & $95 \%$ CI & price & $\begin{array}{c}\text { abs. } \\
\text { error }\end{array}$ & $\begin{array}{c}\text { rel. } \\
\text { error }(\%)\end{array}$ & $\begin{array}{c}\text { time } \\
(\text { sec.) }\end{array}$ \\
\hline 5 & 7 & $(10.3137,2.1 \mathrm{e}-3)$ & {$[10.3097,10.3179]$} & 10.3151 & $1.2 \mathrm{e}-03$ & $\approx 0.01$ & 1.05 \\
8 & 7 & $(10.6662,2.1 \mathrm{e}-3)$ & {$[10.6594,10.6675]$} & 10.6647 & $1.5 \mathrm{e}-03$ & $\approx 0.01$ & 2.88 \\
10 & 7 & $(10.5071,2.1 \mathrm{e}-3)$ & {$[10.5031,10.5112]$} & 10.5055 & $1.6 \mathrm{e}-03$ & $\approx 0.02$ & 4.38 \\
\hline
\end{tabular}

(b) Put options prices, the benchmark prices obtained by the anti-mlMC method $\left(\mathrm{RMSE}=3 \times 10^{-03}\right)$

Table 8.6: European call and put option prices under the jump-extended Heston-1CIR dynamics with parameters from Table 8.2 , "combined-density" treatment, $N_{J}=9, N_{I}=15$, and $10^{-02}$ tolerance for estimating the support of $f_{c}(\cdot)$.

The remaining parameters are similar to those in Table 8.2. In this experiment with the jump-extended model, we use $N_{J}=8$ in (6.16). In these tests, similar to previous tests, the level of resolution is the first $m$ such that $\epsilon_{p}^{(m)}<10^{-02}$, which give $m=7$.

\begin{tabular}{|c|c|c|c|c|c|c|c|c|}
\hline$r_{d}$ & $\begin{array}{c}X_{1}(0) \\
0.02516\end{array}$ & $\begin{array}{c}\kappa_{d_{1}} \\
1.8341\end{array}$ & $\begin{array}{c}\theta_{d_{1}} \\
0.05148\end{array}$ & $\begin{array}{c}\sigma_{d_{1}} \\
0.1543\end{array}$ & $\begin{array}{c}X_{2}(0) \\
0.040016\end{array}$ & $\begin{array}{c}\kappa_{d_{2}} \\
0.005212\end{array}$ & $\begin{array}{c}\theta_{d_{2}} \\
0.03083\end{array}$ & $\begin{array}{c}\sigma_{d_{2}} \\
0.06689\end{array}$ \\
\hline$r_{f}$ & $\begin{array}{c}Y_{1}(0) \\
0.02638\end{array}$ & $\begin{array}{c}\kappa_{f_{1}} \\
1.5446\end{array}$ & $\begin{array}{c}\theta_{f_{1}} \\
0.02638\end{array}$ & $\begin{array}{c}\sigma_{f_{1}} \\
0.08515\end{array}$ & $\begin{array}{c}Y_{2}(0) \\
0.02120\end{array}$ & $\begin{array}{c}\kappa_{f_{2}} \\
0.01265\end{array}$ & $\begin{array}{c}\theta_{f_{2}} \\
0.02120\end{array}$ & $\begin{array}{c}\sigma_{f_{2}} \\
0.04579\end{array}$ \\
\hline $\begin{array}{c}j \\
\text { Set } 1 \\
\nu\end{array}$ & $\begin{array}{c}\nu(0) \\
0.0275\end{array}$ & $\begin{array}{c}\kappa_{\nu} \\
1.70\end{array}$ & $\begin{array}{c}\bar{\nu} \\
0.0232\end{array}$ & $\begin{array}{c}\sigma_{\nu} \\
0.1500\end{array}$ & & & & \\
\hline $\begin{array}{c}\text { Set } 2 \\
\nu\end{array}$ & $\begin{array}{c}\nu(0) \\
0.2\end{array}$ & $\begin{array}{l}\kappa_{\nu} \\
0.1\end{array}$ & $\begin{array}{c}\bar{\nu} \\
0.6\end{array}$ & $\begin{array}{l}\sigma_{\nu} \\
0.5\end{array}$ & & & & \\
\hline jump & $\begin{array}{c}\lambda \\
0.2\end{array}$ & $\begin{array}{c}\tilde{\mu} \\
-0.08\end{array}$ & $\begin{array}{c}\tilde{\sigma} \\
0.3\end{array}$ & & & & & \\
\hline others & $\begin{array}{l}S(0) \\
100\end{array}$ & $\begin{array}{c}K \\
100\end{array}$ & $\begin{array}{l}\rho_{S, \nu} \\
-0.1\end{array}$ & & & & & \\
\hline
\end{tabular}

Table 8.7: Parameters for experiments with the Heston-2CIR models.

In Table 8.8, we present selected pricing results of a European call option. We again observe that all prices computed by the Shannon wavelet method lie within the $95 \%$ confidence intervals obtained with the anti-mlMC method. Moreover, they are in excellent agreement with the benchmark prices, regardless of whether or not the Feller condition is satisfied. We also note the significant efficiency of the Shannon wavelet method.

We conclude this section by noting two points regarding all above experiments. Firstly, while the prices obtained by the proposed Shannon wavelet and the anti-mlMC methods clearly agree, the latter method typically requires from one to two orders of more computational times than the former does, with the most significant difference when the Feller's condition is not satisfied. Secondly, although we do not present respective results obtained by the COS method of Fang and Oosterlee (2008), we note that the COS method is less robust than the SWIFT method in recovering the densities. In particular, for SWIFT, we have a control of the error, via the level of resolution parameter $m$, which 


\begin{tabular}{|c|c|c|c|c|c|c|c|}
\cline { 2 - 8 } \multicolumn{1}{c|}{} & $\begin{array}{c}\nu \\
\text { param. }\end{array}$ & \multicolumn{2}{c|}{ anti-mlMC } & \multicolumn{4}{c|}{ Shannon wavelets } \\
\cline { 3 - 8 } \multicolumn{1}{c|}{} & & (price, std. dev. $)$ & $95 \%$ CI & price & $\begin{array}{c}\text { abs. } \\
\text { error }\end{array}$ & $\begin{array}{c}\text { rel. } \\
\text { error }(\%)\end{array}$ & $\begin{array}{c}\text { time } \\
\text { (sec. })\end{array}$ \\
\hline & Set 1 & $(14.4405,2.1 \mathrm{e}-03)$ & {$[14.4364,14.4446]$} & 14.4407 & $2.5 \mathrm{e}-04$ & $<0.01$ & 0.17 \\
Heston-CIR & Set 2 & $(30.1924,2.1 \mathrm{e}-03)$ & {$[30.1882,30.1965]$} & 30.1922 & $2.2 \mathrm{e}-04$ & $<0.01$ & 0.17 \\
\hline jump-ext. & Set 1 & $(16.7533,2.1 \mathrm{e}-03)$ & {$[16.7492,16.7575]$} & 16.7529 & $3.8 \mathrm{e}-04$ & $<0.01$ & 0.95 \\
Heston-CIR & Set 2 & $(31.2892,2.1 \mathrm{e}-03)$ & {$[31.2850,31.2934]$} & 31.2888 & $4.1 \mathrm{e}-04$ & $<0.01$ & 0.92 \\
\hline
\end{tabular}

Table 8.8: European call option prices under Heston-2CIR dynamics with $T=3$ using parameters from Table 8.7, "combined-density" treatment, $N_{J}=9, N_{I}=15$, and $10^{-02}$ tolerance for estimating the support of $f_{c}(\cdot)$. For the anti-mlMC method, the RMSE is set to $3 \times 10^{-3}$.

does not rely on a priori truncation of the integration domain, as opposed to trial-and-error in the COS method, which changes the integration domain, and hence affects its accuracy.

\section{Conclusions and future work}

In this paper, we extend the applicabilities of existing state-of-the-art numerical integration methods to the broad class of jump-extended Heston models with multi-factor CIR interest rate dynamics. While we focus on the SWIFT of Ortiz-Gracia and Oosterlee (2016), due to its established robustness, the results presented in this paper can be easily extended to the COS method of Fang and Oosterlee (2008) as well.

Traditionally, a direct application of these integration methods require knowing a closed-form expression for the characteristic function of the underlying process, which is not available for this general class of models. We show that within the Monte-Carlo and PDE hybrid computational framework put forward in Dang et al. (2015b, 2017), it is possible to develop a very robust and highly efficient pricing numerical integration technique for these models. In particular, the proposed drSWIFT method enjoys a significant dimension reduction, from two multi-factor interest rate processes to only a one-factor process. As such, the computational complexity of drSWIFT method is independent of the number of stochastic factors in the model. Although in this work, we primarily focus on FX options, the proposed model and computational method can be easily utilized or adapted to European options in other markets, such as equity.

Regarding future work, we particularly emphasize the potential of the hybrid MC and PDE computational approach in general, and of the drSWIFT method in particular, for problems that require significant computational power. An example of such a problem is model calibration which involves the pricing of a wide range of options. In this case, the proposed methodology could be useful, because of its excellent speed, accuracy, and robustness. Another example is the computation of valuation adjustments (xVA) for over-the-counter financial derivatives (Feng et al., 2016; Graaf et al., 2014; Gregory, 2012, 2015; Karlsson et al., 2016). Preliminary results indicate that the hybrid MC and PDE computational approach combined with Shannon wavelets result in efficient computation of exposure profiles for counter-party credit risk in the context of the early exercise features.

\section{References}

Ahlip, R., L. A. F. Park, and A. Prodan (2017). Pricing currency options in the Heston/CIR double exponential jump-diffusion model. International Journal of Financial Engineering 4(1), 1-30.

Ahlip, R. and M. Rutkowski (2013). Pricing of foreign exchange options under the Heston stochastic volatility model and CIR interest rates. Quantitative Finance 13, 955-966. 
Ahlip, R. and M. Rutkowski (2015). Semi-analytical pricing of currency options in the Heston/CIR jump-diffusion hybrid model. Applied Mathematical Finance 22(1), 1-27.

Amin, H. H. N. (2012). Calibration of different interest rate models for a good fit of yield curves. Master's thesis, Delft University of Technology.

Andersen, L. and V. Piterbarg (2007). Moment explosions in stochastic volatility models. Finance and Stochastics 11, 29-50.

Brigo, D. and F. Mercurio (2006). Interest Rate Models - Theory and Practice. Springer, second edition.

Broadie, M. and O. Kaya (2006). Exact simulation of stochastic volatility and other affine jump difusion processes. Operations Research 54, 217-231.

Caps, O. (2007). On the valuation of power-reverse duals and equity-rates hybrids. Presented at Frankfurt MathFinance Conference, Derivatives and Risk Management in Theory and Practice.

Cattani, C. (2008). Shannon wavelets theory. Mathematical Problems in Engineering 2008, article ID 164808.

Chen, R.-R. and L. Scott (1992). Pricing interest rate options in a two-factor Cox-Ingersoll-Ross model of the term structure. The Review of Financial Studies 5, 613-636.

Chen, R.-R. and L. Scott (2003). Multi-factor Cox-Ingersoll-Ross models of the term structure: Estimates and tests from a Kalman filter model. The Journal of Real Estate Finance and Economics $27,143-172$.

Clark, I. J. (2011). Foreign Exchange Option Pricing. John Wiley \& Sons, Inc, first edition.

Col, A. D., A. Gnoatto, and M. Grasselli (2013). Smiles all around: FX joint calibration in a multiHeston model. Journal of Banking \& Finance 37, 3799-3818.

Colldeforns-Papiol, G., L. Ortiz-Gracia, and C. W. Oosterlee (2017). Two-dimensional Shannon wavelet inverse Fourier technique for pricing European options. Applied Numerical Mathematics $117,115-138$.

Cont, R. and P. Tankov (2004). Financial Modelling with Jump Processes. Chapman and Hall.

Cox, J., J. Ingersoll, and S. Ross (1985a). A theory of the term structure of interest rates. Econometrica $53,385-407$.

Cox, J. C., J. E. Ingersoll, and S. A. Ross (1985b). A theory of the term structure of interest rates. Econometrica 53, 385-407.

Cozma, A., M. Mariapragassam, and C. Reisinger. (2018). Convergence of an Euler discretisation scheme for the Heston stochastic-local volatility model with CIR interest rates. SIAM Journal on Financial Mathematics 9, 127-170.

Cozma, A. and C. Reisinger (2017). A mixed Monte Carlo and PDE variance reduction method for foreign exchange options under the Heston-CIR model. Journal of Computational Finance 20, $109-149$.

Cui, Y., S. del Bano Rollin, and G. Germano (2017). Full and fast calibration of the Heston stochastic volatility model. European Journal of Operational Research 263, 625-638. 
Dang, D. M. (2017). A multi-level dimension reduction Monte-Carlo method for jump-diffusion models. Journal of Computational and Applied Mathematics 324, 49-71.

Dang, D. M., C. Christara, and K. Jackson (2014). GPU pricing of exotic cross-currency interest rate derivatives with a foreign exchange volatility skew model. Journal of Concurrency and Computation: Practice and Experience 26, 1609-1625.

Dang, D. M., C. Christara, K. Jackson, and A. Lakhany (2010). A PDE pricing framework for cross-currency interest rate derivatives. In Proceedings of the 10th International Conference in Computational Science (ICCS), volume 1, pp. 2371-2380. Procedia Computer Science.

Dang, D. M., C. Christara, K. Jackson, and A. Lakhany (2015a). An efficient numerical PDE approach for pricing foreign exchange interest rate hybrid derivatives. Journal of Computational Finance 18, $1-55$.

Dang, D. M., K. R. Jackson, and M. Mohammadi (2015b). Dimension and variance reduction for Monte-Carlo methods for high-dimensional models in finance. Applied Mathematical Finance 22, $522-552$.

Dang, D. M., K. R. Jackson, and S. Sues (2017). A dimension and variance reduction Monte-Carlo method for pricing and hedging options under jump-diffusion models. Applied Mathematical Finance $24,175-215$.

Dang, D. M. and L. Ortiz-Gracia (2018). A dimension reduction Shannon-wavelet based method for option pricing. Journal of Scientific Computing 75, 733-761.

Delbaen, F. and W. Schachermayer (1994). A general version of the fundamental theorem of asset pricing. Mathematische Annalen 300, 463-520.

Driffill, J., T. Kenc, and M. Sola (2003). An empirical examination of term structure models with regime shifts. Computing in Economics and Finance, Society for Computational Economics .

Dufresne, D. (2001). The integrated square-root process pp. 1-22. Working paper No.90, Centre for Actuarial Studies, University of Melbourne.

Erismann, M. (2011). Analytical propositions to evaluate contingent convertible capital. Master's thesis, University of St. Gallen.

Fang, F. and C. W. Oosterlee (2008). A novel pricing method for European options based on FourierCosine series expansions. SIAM Journal on Scientific Computing 31, 826-848.

Fang, F. and C. W. Oosterlee (2011). A Fourier-based valuation method for Bermudan and barrier options under Heston's model. SIAM Journal on Financial Mathematics 2, 439-463.

Feng, Q., S. Jain, P. Karlsson, B. D. Kandhai, and C. Oosterlee (2016). Effcient computation of exposure profiles on real-world and risk-neutral scenarios for Bermudan swaptions. Journal of Computational Finance 20, 139-172.

Garman, M. B. and S. W. Kohlhagen (1983). Foreign currency option values. Journal of International Money and Finance 2, 231-237.

Giles, M. B. (2008). Multi-level Monte Carlo path simulation. Operations Research 56, 607-617.

Giles, M. B. and L. Szpruch (2014). Antithetic multilevel Monte Carlo esitmation for multi-dimensional SDEs without lévy area simulation. The Annals of Applied Probability 24, 1585-1620. 
Graaf, C., Q. Feng, B. Kandhai, and C. Oosterlee (2014). Efficient computation of exposure profiles for counterparty credit risk. International Journal of Theoretical and Applied Finance 17, 1450024-1.

Gregory, J. (2012). Counterparty Credit Risk and Credit value adjustment. John Wiley \& Sons, Ltd, second edition.

Gregory, J. (2015). The xVA Challenge. John Wiley \& Sons, Ltd, first edition.

Heston, S. (1993). A closed form solution for options with stochastic volatility with applications to bond and currency options. Review of Financial Studies 6, 327-343.

Hull, J. and A. White (1993). One factor interest rate models and the valuation of interest rate derivative securities. Journal of Financial and Quantitative Analysis 28(2), 235-254.

Jamshidian, F. and Y. Zhu (1997). Scenario Simulation: Theory and methodology. Finance and Stochastic 13, 43-67.

Karlsson, P., S. Jain, and C. W. Oosterlee (2016). Counterparty credit exposures for interest rate derivatives using the Stochastic Grid Bundling Method. Applied Mathematical Finance 23, 175-196.

Kou, S. G. (2002). A jump diffusion model for option pricing. Management Science 48, 1086-1101.

Lafférs, L. (2009). Empirical likelihood estimation of interest rate diffusion model. Master's thesis, Comenius University in Bratislava.

Mallo, C. (2010). Turnover of the global foreign exchange markets in April 2010. Triennial Central Bank Survey of Foreign Exchange and Derivatives Market Activity.

Maree, S. C., L. Ortiz-Gracia, and C. W. Oosterlee (2017). Pricing early-exercise and discrete barrier options by Shannon wavelet expansions. Numerische Mathematik 136(4), 1035-1070.

Merton, R. (1976). Option pricing when underlying stock returns are discontinuous. Journal of Financial Economics 3, 125-144.

Nawalkha, S. K., N. A. Beliaeva, and G. M. Soto (2007). Dynamic Term Structure Modeling: The Fixed Income Valuation Course. John Wiley \& Sons, Inc.

Neuenkirch, A. and L. Szpruch (2014). First order strong approximations of scalar SDEs with values in a domain. Numerische Mathematik 128, 103-136.

Ortiz-Gracia, L. and C. W. Oosterlee (2016). A highly efficient Shannon wavelet inverse Fourier technique for pricing European options. SIAM Journal on Scientific Computing 38(1), B118-B143.

Piterbarg, V. (2006). Smiling hybrids. Risk magazine 19(5), 66-70.

Qu, D. (2016). Manufacturing and Managing Customer-Driven Derivatives. John Wiley \& Sons, Inc, first edition.

Rebonato, R. (1998). Interest Rate Option Models. John Wiley \& Sons, Inc, second edition.

Sippel, J. and S. Ohkoshi (2002). All power to PRDC notes. Risk magazine 15(11), 1-3.

Stenger, F. (2011). Handbook of Sinc Numerical Methods. CRC Press. 